ARTICLE

https://doi.org/10.1038/s41467-019-08567-6

\title{
Reversible fold-switching controls the functional cycle of the antitermination factor $\mathrm{RfaH}$
}

\author{
Philipp Konrad Zuber ${ }^{1}$, Kristian Schweimer ${ }^{1}$, Paul Rösch ${ }^{1,2}$, Irina Artsimovitch ${ }^{3,4} \&$ Stefan H. Knauer (i) ${ }^{1}$
}

$\mathrm{RfaH}$, member of the NusG/Spt5 family, activates virulence genes in Gram-negative pathogens. RfaH exists in two states, with its C-terminal domain (CTD) folded either as $\alpha$-helical hairpin or $\beta$-barrel. In free $\mathrm{RfaH}$, the $\alpha$-helical CTD interacts with, and masks the RNA polymerase binding site on, the $\mathrm{N}$-terminal domain, autoinhibiting $\mathrm{RfaH}$ and restricting its recruitment to opsDNA sequences. Upon activation, the domains separate and the CTD refolds into the $\beta$-barrel, which recruits a ribosome, activating translation. Using NMR spectroscopy, we show that only a complete ops-paused transcription elongation complex activates $\mathrm{RfaH}$, probably via a transient encounter complex, allowing the refolded CTD to bind ribosomal protein S10. We also demonstrate that upon release from the elongation complex, the CTD transforms back into the autoinhibitory $\alpha$-state, resetting the cycle. Transformation-coupled autoinhibition allows RfaH to achieve high specificity and potent activation of gene expression.

\footnotetext{
${ }^{1}$ Lehrstuhl Biopolymere, Universität Bayreuth, Universitätsstraße 30, 95447 Bayreuth, Germany. ${ }^{2}$ Forschungszentrum für Bio-Makromoleküle, Universität Bayreuth, Universitätsstraße 30, 95447 Bayreuth, Germany. ${ }^{3}$ Department of Microbiology, The Ohio State University, Columbus, OH 43210, USA. ${ }^{4}$ The Center for RNA Biology, The Ohio State University, Columbus, OH 43210, USA. Correspondence and requests for materials should be addressed to

I.A. (email: artsimovitch.1@osu.edu) or to S.H.K. (email: stefan.knauer@uni-bayreuth.de)
} 
M ulti-subunit RNA polymerases (RNAP) transcribe all cellular genomes and interact with a plethora of accessory proteins that modulate every step of RNA synthesis. Among them, NusG/Spt5 is the only regulator that is conserved across all domains of life ${ }^{1}$. NusG homologs control gene expression by reducing RNAP pausing and arrest to enhance its processivity ${ }^{2-4}$ and by enabling crosstalk between transcription and concomitant cellular processes. These proteins physically link elongating RNAP to a ribosome $\mathrm{e}^{5}$ or transcription termination factor Rho ${ }^{6,7}$ in bacteria and to factors mediating mRNA capping $^{8}$, histone modification ${ }^{9}$, and somatic hypermutation ${ }^{10}$ in eukaryotes.

The modular structure of NusG proteins underpins this bridging activity (Fig. 1a). The N-terminal domains (NTDs) exhibit mixed $\alpha / \beta$ topology and establish similar contacts to the two largest subunits of bacterial, archaeal, and eukaryotic RNAPs $^{11-15}$. In bacteria, these contacts are mediated by the $\beta^{\prime}$ clamp and the $\beta$ lobe and protrusion domains ${ }^{13}$. The $\mathrm{C}$-terminal domains (CTDs; one in bacteria and archaea, multiple in eukaryotes) contain a Kyrpides, Ouzounis, Woese motif ${ }^{16}$, fold into a five-stranded $\beta$-barrel that is flexibly connected to the NTD, and serve as interaction platform for various binding partners, making co-transcriptional contacts to cellular proteins that ultimately determine their effects on gene expression. In Escherichia coli, NusG-CTD interacts with Rho to inhibit synthesis of foreign and aberrant RNAs ${ }^{6,17}$ or with ribosome to couple transcription to translation ${ }^{5,18}$.

In addition to housekeeping factors that co-localize with elongating RNAP across most genes ${ }^{19-21}$, highly specialized NusG paralogs are present in ciliates ${ }^{22}$, plants ${ }^{23}$, and bacteria ${ }^{24}$. In order to avoid off-target recruitment, these paralogs must be specifically recruited to their target genes. This is particularly critical when their function is opposite to that of housekeeping NusG, as is the case of bacterial paralogs which silence Rhodependent termination ${ }^{25}$.

$\mathrm{RfaH}$, the best studied NusG paralog, activates expression of cell wall biosynthesis, conjugation, and virulence genes by inhibiting Rho ${ }^{26}$; mutations in rho and nus $G$ suppress the loss of $E$. coli $r f \mathrm{H}^{27}$. $\mathrm{RfaH}$ activates fewer than ten operons in E. coli, each containing an operon polarity suppressor (ops) element in their $5^{\prime}$ $\mathrm{UTRs}^{28}$. When RNAP pauses at the ops site, the non-template (NT) DNA strand in the transcription bubble forms a hairpin structure $^{13,29}$. During recruitment, $\mathrm{RfaH}$ makes base-specific contacts with two flipped-out ops bases via its NTD. While these contacts explain sequence specificity of $\mathrm{RfaH}$, off-target recruitment and competition with housekeeing NusG is additionally controlled by autoinhibition.
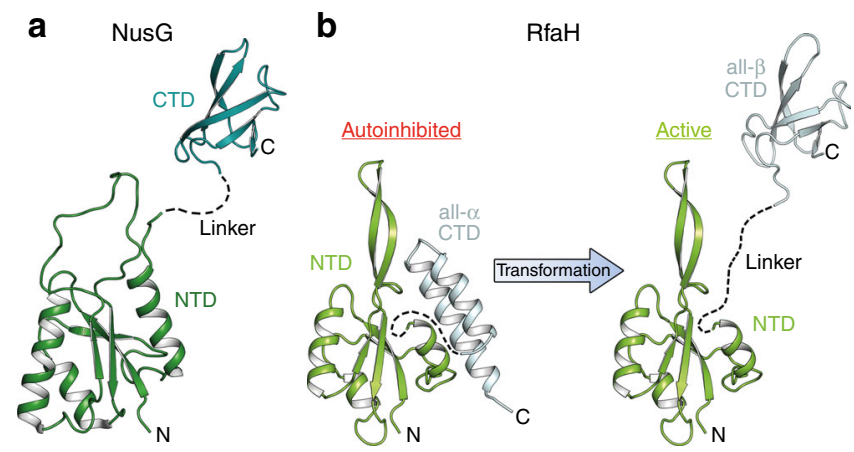

Fig. 1 Structures of NusG and RfaH from E. coli. Structures of a NusG and $\mathbf{b} \mathrm{RfaH}$ are in ribbon representation, the linker connecting the domains is indicated by a dashed line. PDB IDs: NusG-NTD, '2K06'; NusG-CTD, '2JVV'; RfaH, '5OND'; RfaH-CTD, '2LCL'
RfaH-NTD exhibits the mixed $\alpha / \beta$ topology typical for NusG proteins but, in contrast to all other known NusGs, the RfaHCTD folds as an $\alpha$-helical hairpin in free $\mathrm{RfaH}$ (all- $\alpha$ state; Fig. 1b). The CTD hairpin tightly interacts with the NTD, masking the RNAP-binding site and autoinhibiting $\mathrm{RfaH}^{30}$. The relief of autoinhibition requires domain dissociation, thought to be triggered by transient contacts to ops. Thereafter, the released NTD binds to the clamp helices of the $\beta^{\prime}$ subunit $\left(\beta^{\prime} \mathrm{CH}\right)$ and the gate loop of the $\beta$ subunit ( $\beta \mathrm{GL}$ ) of the RNAP 26,28 , while the CTD spontaneously and completely refolds into a NusG-like $\beta$-barrel (all- $\beta$ state; Fig. $1 \mathrm{~b}$ ) and recruits the ribosome via interactions with ribosomal protein S10, substituting for a missing ShineDalgarno sequence ${ }^{31}$. As striking as this transformation is, the lack of spurious $\mathrm{RfaH}$ recruitment at non-ops sites ${ }^{28}$ suggests that refolding may be reversible: following dissociation from RNAP at a terminator, $\mathrm{RfaH}$ must either perish or transform back into the autoinhibited state ${ }^{32}$ because activated $\mathrm{RfaH}$ does not require ops for recruitment ${ }^{30,33}$

Here, we used NMR spectroscopy adapted to supramolecular, multicomponent systems in combination with functional studies to explore the conformational transitions that accompany RfaH binding to and dissociation from RNAP. Our results indicate that $\mathrm{RfaH}$ functions in a true cycle. We identify the ops-paused transcription elongation complex (EC) as a minimal activation signal for $\mathrm{RfaH}$ and demonstrate that, upon recruitment, $\mathrm{RfaH}-$ CTD refolds into the $\beta$-barrel that subsequently interacts with ribosomal protein S10. We further show that RfaH-CTD refolds into the $\alpha$-helical state after RfaH release from the EC, thereby reestablishing the autoinhibited state. Our results demonstrate unmatched conformational and functional plasticity of $\mathrm{RfaH}$, which refolds not once but twice during its functional cycle, as befits a transformer protein ${ }^{34}$.

\section{Results}

The ops-paused EC is necessary for RfaH activation. To elucidate the mechanism of $\mathrm{RfaH}$ recruitment to the $\mathrm{EC}$ and identify a signal that induces domain opening, we used a combination of solution-state NMR spectroscopy approaches which allow the characterization of protein:ligand interactions and structural transitions as well as the analysis of excited, low-populated states. In addition to uniformly ${ }^{15} \mathrm{~N}$-labeled proteins, samples were employed where $\left[{ }^{1} \mathrm{H},{ }^{13} \mathrm{C}\right]$-labeled methyl groups of Ile, Leu, and Val residues in perdeuterated proteins served as NMR-active probes ([I,L,V]-labeling); the latter method increases the sensitivity to enable studies of large complexes ${ }^{35}$.

In the methyl-transverse relaxation optimized spectroscopy (methyl-TROSY) spectrum of free [I,L,V]-RfaH we observed only signals of the autoinhibited form (Fig. 2a). To test if, nevertheless, free $\mathrm{RfaH}$ exists in equilibrium of the closed and open conformations, with the open state being only low-populated, we first carried out ${ }^{15} \mathrm{~N}$-based chemical exchange saturation transfer (CEST) experiments (Supplementary Figure 1a-d; ref. ${ }^{36}$ ). This method allows for the detection and characterization of 'invisible', i.e., sparsely populated, excited conformational states that are in slow chemical exchange with a visible ground-state conformation. In CEST experiments the saturation of ${ }^{15} \mathrm{~N}$ spins by a weak radio frequency field can be transferred between different conformational states if these states exchange on a timescale of 5-50 ms. If the transmitter frequency, which is stepped through the spectral region of ${ }^{15} \mathrm{~N}$ spins, coincides with the resonance frequency of a spin, the signal intensity is significantly decreased, causing a dip in the CEST profile (normalized intensity $\left(I / I_{0}\right)$ of a signal as function of the transmitter frequency of the saturation field). If the major state is in equilibrium with another (minor) state, the exchange 


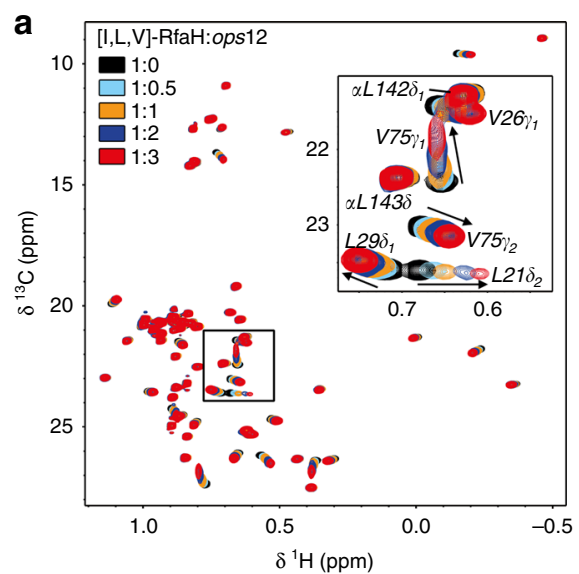

b

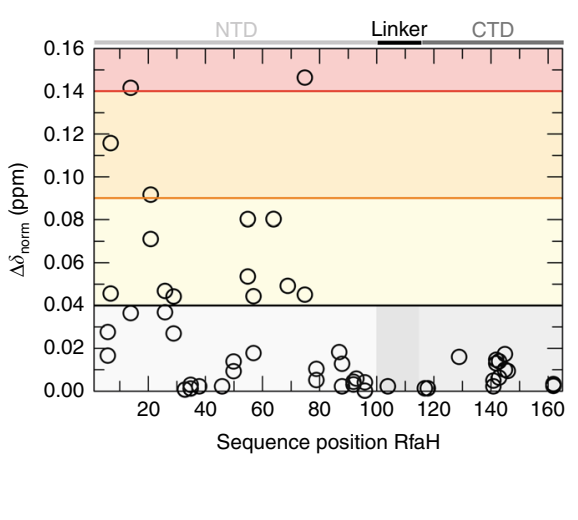

c

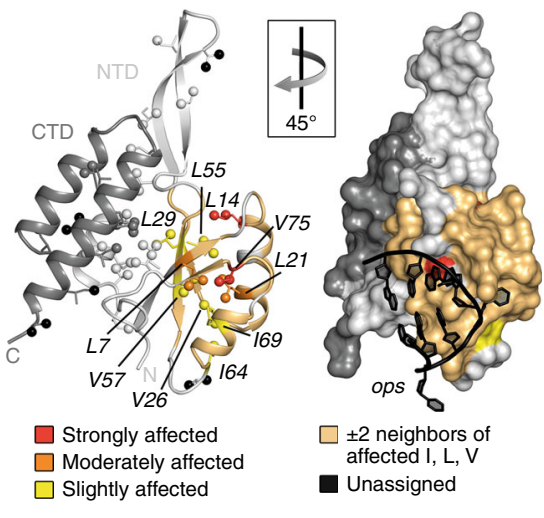

Fig. 2 RfaH:ops interaction. a 2D $\left[{ }^{1} \mathrm{H},{ }^{13} \mathrm{C}\right]$ methyl-TROSY spectra of $45 \mu \mathrm{M}[\mathrm{I}, \mathrm{L}, \mathrm{V}]-\mathrm{RfaH}$ titrated with ops (concentration of stock solution: $1.3 \mathrm{mM}$ ). Inset: enlargement of boxed region. $\mathbf{b}$ Interaction of [I,L,V]-RfaH with ops. Methyl-TROSY-derived normalized chemical shift changes vs. sequence position of $\mathrm{RfaH}$ (corresponding spectra are depicted in a). Horizontal lines: significance levels of $\Delta \delta_{\text {norm }}=0.04 \mathrm{ppm}$, black; $=0.09 \mathrm{ppm}$, orange, $=0.14 \mathrm{ppm}$, red. Source data are provided as a Source Data file. c ops binding surface of RfaH as derived from the titration of [I,L,V]-RfaH with ops. Affected methyl groups are mapped onto the RfaH:ops9 structure (PDB ID: '5OND'). RfaH is shown in ribbon (left) and surface (right) representation (RfaH-NTD, light gray; RfaHCTD, dark gray), ops9 in ribbon representation (black) with nucleosides as sticks. The arrow indicates how the structures are rotated with respect to each other. Termini are labeled. For graphical representation of the interaction site the whole amino acid is colored. Ile, Leu, and Val residues are shown as sticks with the carbon atoms of the methyl groups as spheres. Slightly affected $\left(0.04 \leq \Delta \delta_{\text {norm }}<0.09 \mathrm{ppm}\right)$, yellow; moderately affected $\left(0.09 \leq \Delta \delta_{\text {norm }}<0.14\right.$ $\mathrm{ppm})$, orange; strongly affected $\left(\Delta \delta_{\text {norm }} \geq 0.14 \mathrm{ppm}\right)$, red; unaffected, colored according to their domain; not assigned methyl groups, black. Two amino acids on either side of an affected Ile/Leu/Val residue are highlighted in beige unless they were unaffected Ile/Leu/Val residues

between these states will be evidenced by a second dip in the CEST profile occurring at the resonance frequency of the minor state. In the CEST profiles of the RfaH-CTD residues that have well-separated signals in the all- $\alpha$ and the all- $\beta$ state, no second dip at the expected chemical-shift position corresponding to the all $-\beta$ state could be observed (Supplementary Figure 1a-d). Thus, within the detection limits of the CEST experiment (population > $0.2 \%$, exchange rate $20-200 \mathrm{~s}^{-1}$ ), all free $\mathrm{RfaH}$ occupies the closed, autoinhibited state.

To test whether RfaH opens and closes on a timescale faster than accessible by CEST experiments, we performed CarrPurcell-Meiboom-Gill sequence (CPMG) experiments. This analysis enables the measurement of the contribution of chemical exchange to the transverse relaxation rate $\left(R_{2}\right)$ of any nucleus for exchange processes in the range of $\sim 200-2000 \mathrm{~s}^{-137}$. In brief, a series of refocusing $180^{\circ}$ pulses is applied with different time intervals $\left(\tau_{180}\right)$ between the pulses. During long time intervals, i.e., at low CPMG frequencies $\left(1 / 2 \tau_{180}\right)$, the chemical exchange can contribute to $R_{2}$, resulting in an increase of $R_{2}$. In the CMPG experiments of ${ }^{15} \mathrm{~N}$-RfaH, we observed slightly enhanced $R_{2}$ rates at lower CPMG frequencies for residues located in a loop in the DNA-binding region ${ }^{29}$ (T72, V75) as well as in the $\beta$-hairpin (S47), suggesting that these RfaH-NTD regions exhibit flexibility (Supplementary Figure 1e). In contrast, $R_{2 \text {,eff }}$ did not change for $\mathrm{RfaH}-\mathrm{NTD}$ residues in the domain interface (F51) or RfaH-CTD residues (F123, G135, M140, L145; Supplementary Figure 1e), indicating that the autoinhibited state is stable. Together, these findings argue against an equilibrium of the closed and open conformations of $\mathrm{RfaH}$ on a timescale faster than $0.5 \mathrm{~ms}-50 \mathrm{~ms}$.

The DNA-binding site of RfaH is located on the RfaH-NTD, opposite the RfaH-CTD interaction surface ${ }^{13,29}$. A $\left[{ }^{1} \mathrm{H},{ }^{15} \mathrm{~N}\right]-$ heteronuclear single quantum coherence (HSQC)-based NMR titration of ${ }^{15} \mathrm{~N}$-RfaH with ops indicated that binding of RfaH to opsDNA does not induce domain separation ${ }^{29}$. Exploiting the high sensitivity of methyl groups, we next wanted to corroborate this result. Chemical-shift changes upon titration of [I,L,V]-RfaH with opsDNA were consistent with the DNA-binding site determined via the ${ }^{15} \mathrm{~N}$-based titration and observed in the
RfaH:ops 9 crystal structure and in the cryo electron microscopy (EM) RfaH:ops-paused EC structure (Fig. 2; refs. ${ }^{13,29}$ ). Signals corresponding to the all- $\beta$ RfaH-CTD could not be observed during the titration, suggesting that binding to DNA alone cannot be a signal for domain opening.

RfaH weakly binds to free RNAP ${ }^{38}$. To test whether these contacts could activate $\mathrm{RfaH}$, we measured one- and twodimensional (1D, 2D) methyl-TROSY spectra of [I,L,V]-RfaH titrated with RNAP (Fig. 3a). The overall intensity of signals corresponding to autoinhibited $\mathrm{RfaH}$ decreased uniformly, but no changes in chemical shifts were observed and $\beta$-barrel CTD signals did not appear (Fig. 3a and Supplementary Figure 2), indicating that while RfaH can interact with RNAP, this binding does not induce domain dissociation/transformation. Adding an excess of NusG-NTD to the [I,L,V]-RfaH:RNAP complex recovered some of the intensity of [I,L,V]-RfaH signals (Fig. 3b), implying RfaH displacement by NusG-NTD. Since RfaH and NusG share binding sites ${ }^{13,28}$, this finding suggests that the closed $\mathrm{RfaH}$ binds near the final RfaH-NTD binding site on the EC.

These results show that neither DNA nor RNAP alone can relieve $\mathrm{RfaH}$ autoinhibition. To test if EC paused at the ops site (opsEC) is suffient to induce domain separation, we assembled $o p s E C$ with a nucleic-acid scaffold (Supplementary Figure 3) and perdeuterated RNAP. A methyl-TROSY-based titration of [I,L, $\mathrm{V}]-\mathrm{RfaH}$ with the opsEC showed that signal intensity of [I,L,V]$\mathrm{RfaH}$ methyl groups decreased non-uniformly, with only slight chemical shift changes (Fig. 4a). Signals of the $\alpha$-helical RfaHCTD disappeared while, concurrently, resonances corresponding to the $\beta$-barrel RfaH-CTD appeared and gradually intensified, indicating refolding of the RfaH-CTD (Fig. 4a and Supplementary Figure 4). Next we wanted to exclude the possibility that the $\mathrm{RfaH}-\mathrm{CTD}$ refolding is due to cleavage of the RfaH linker caused by protease impurities or sample degradation during long-lasting NMR experiments. Both scenarios would lead to the release of the $\mathrm{RfaH}-\mathrm{CTD}$ and its subsequent spontaneous transformation, as shown for an $\mathrm{RfaH}$ variant where a TEV protease cleavage site was introduced into the linker ${ }^{31}$. Thus, we performed translational diffusion experiments of $[\mathrm{I}, \mathrm{L}, \mathrm{V}]-\mathrm{RfaH}$ and $[\mathrm{I}, \mathrm{L}, \mathrm{V}]-\mathrm{RfaH}-$ 


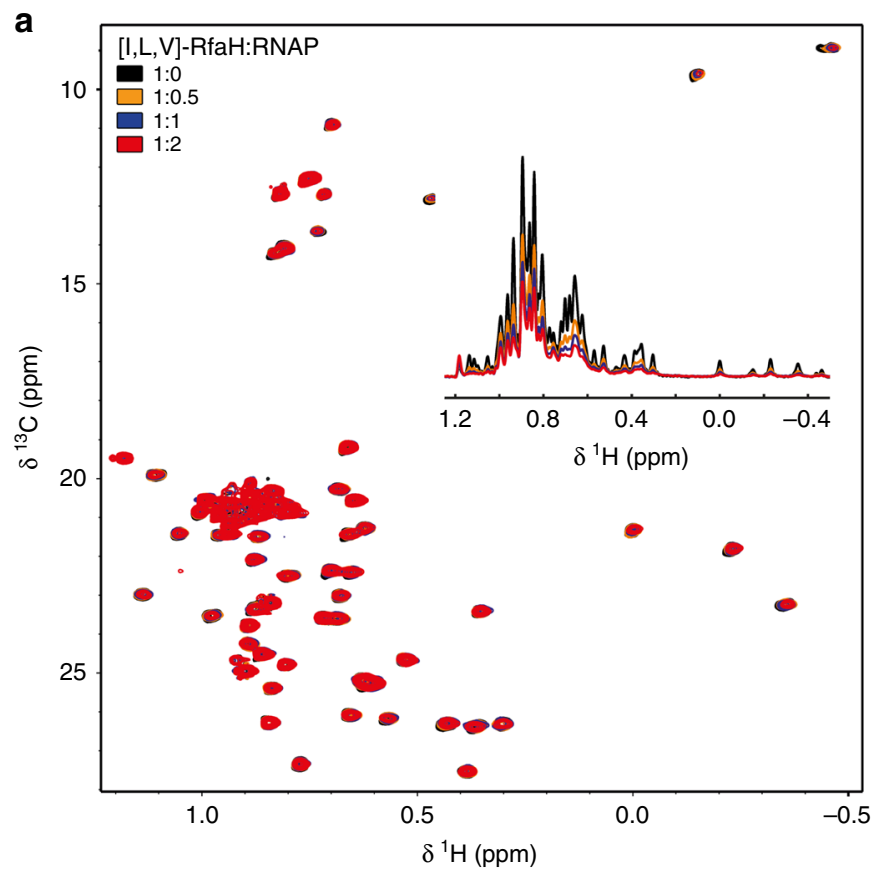

b

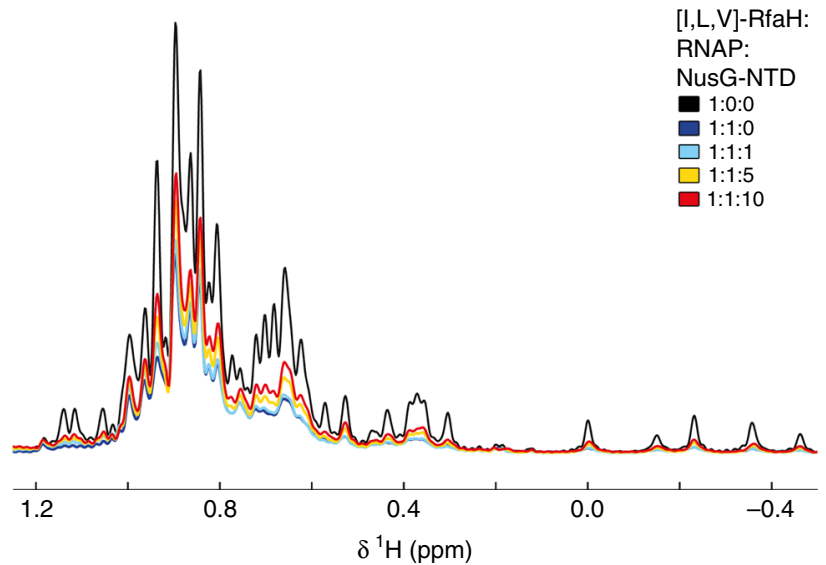

Fig. 3 Binding of RfaH to RNAP. a $2 \mathrm{D}\left[{ }^{1} \mathrm{H},{ }^{13} \mathrm{C}\right]$ methyl-TROSY spectra of $15 \mu \mathrm{M}[\mathrm{I}, \mathrm{L}, \mathrm{V}]-\mathrm{RfaH}$ titrated with RNAP (concentration of stock solution: $78 \mu \mathrm{M})$. Inset: normalized 1D $\left[{ }^{1} \mathrm{H},{ }^{13} \mathrm{C}\right]$-methyl-TROSY spectra, colored as 2D spectra. See also Supplementary Figure 2. b Displacement of RfaH from RNAP by NusG-NTD. Normalized 1D methyl-TROSY spectra of [I,L,V]-RfaH (30 $\mu \mathrm{M}$, black), [I,L,V]-RfaH in the presence of equimolar RNAP $(30 \mu \mathrm{M}$ each, red), and [I,L,V]-RfaH upon titration of the [I,L,V]-RfaH:RNAP complex (30 $\mu \mathrm{M}$ each) with $712 \mu \mathrm{M}$ NusG-NTD; molar ratio of [I,L,V]-RfaH: RNAP:NusG-NTD is indicated in color

CTD in the absence and presence of opsEC (Supplementary Figure 5a-e). The diffusion coefficient of [I,L,V]-RfaH:opsEC is significantly smaller than that of free [I,L,V]-RfaH or [I,L,V]RfaH-CTD (Supplementary Table 1), confirming that the all- $\beta$ signals in the [I,L,V]-RfaH:opsEC sample arise from $\mathrm{RfaH}$ bound to opsEC and that signals of the freed RfaH-CTD are visible even when $\mathrm{RfaH}$ is bound to the opsEC.

The decrease of RfaH-NTD methyl group signal intensity likely is a combination of two effects: a general decrease resulting from the increased molecular mass of $[\mathrm{I}, \mathrm{L}, \mathrm{V}]-\mathrm{RfaH}$ upon complex formation and a non-uniform decrease due to slow or intermediate exchange on the chemical shift timescale. Thus we analyzed the signal intensity in certain titration steps quantitatively to identify affected residues as established ${ }^{39}$ (Fig. 4b). In brief, in each titration step, relative intensity, i.e., the ratio of remaining signal intensity to that in the spectrum of free [I,L,V]$\mathrm{RfaH}$, was determined. Residues with relative signal intensities below certain thresholds were classified as either strongly or moderately affected (for details see Methods section). Mapping of the relative signal intensity of the 1:0.5 complex on the threedimensional structure of RfaH-NTD revealed a patch where signal intensity changed significantly (Fig. $4 c$ ). To aid visualization limited by a small number of NMR-active probes, we graphically extended the representation of affected residues by including the two flanking residues on each side, unless they were an unaffected Ile, Leu, or Val residue (beige in Fig. 4c). Comparing the affected regions with the cryo EM structure of the RfaH:opsEC ${ }^{13}$ shows that the main, high-affinity contacts with the $\beta^{\prime} \mathrm{CH}$ are in good agreement (Fig. 4d). The HTTT motif in helix $\alpha 2$ of $\mathrm{RfaH}$ interacts with the $\beta \mathrm{GL}^{13,26}$, but since this motif lacks NMR-active probes and the closest labeled residues point to the interior of $\mathrm{RfaH}$, no information on these contacts could be obtained (Fig. 4c). These results demonstrate that in the presence of opsEC RfaH domains dissociate, RfaH-NTD binds to the EC, and RfaH-CTD refolds into the $\beta$-state, confirming that the ops-paused EC is the relevant signal for $\mathrm{RfaH}$ recruitment.

EC-bound RfaH interacts with S10. In a subpopulation of the RfaH:opsEC complexes observed by cryo EM, the RfaH-CTD binds to the $\beta$-flap tip helix at the RNA exit channel ${ }^{13}$. To test if this interaction also occurs in solution, we performed a titration of [I,L,V]-RfaH-CTD with opsEC (Supplementary Figure 5f). In the $1 \mathrm{D}$ methyl-TROSY spectra signal intensity of $[\mathrm{I}, \mathrm{L}, \mathrm{V}]-\mathrm{RfaH}-$ CTD decreases by $\sim 25 \%$ upon addition of opsEC. This loss of intensity indicates complex formation as the molecular mass of [I, $\mathrm{L}, \mathrm{V}]-\mathrm{RfaH}-\mathrm{CTD}$ increases upon opsEC binding, although this interaction might be weak. These observations are in agreement with the finding that the diffusion coefficient of [I,L,V]-RfaHCTD is slightly decreased in the presence of opsEC (Supplementary Table 1). The finding that the signals of the all- $\beta$ RfaHCTD, i.e., the freed CTD, are visible when $\mathrm{RfaH}$ is bound to the opsEC strengthens the hypothesis that RfaH-CTD is only transiently bound to RNAP in the EC.

We argued that $\mathrm{RfaH}$ recruits a ribosome via interactions observed in a binary complex of isolated RfaH-CTD and $S 10^{31}$. To test if this contact is preserved when $\mathrm{RfaH}$ is bound to the $o p s \mathrm{EC}$, we performed an NMR-based titration of [I,L,V]-RfaH with S10 in the presence of the opsEC using S10 lacking the ribosome-binding loop $\left(\mathrm{S}^{\circ} 0^{\Delta}\right)$ in complex with NusB to increase stability ${ }^{40}$. Upon addition of protonated opsEC to [I,L,V]-RfaH in equimolar concentration, mainly signals of the $\beta$-barrel CTD were observable, showing that $\mathrm{RfaH}$ is bound to the opsEC and that the CTD is in the all- $\beta$ state (Fig. 5a). Subsequent titration with $S 10^{\Delta}$ :NusB decreased intensity of some of these signals significantly (Fig. 5a, b). Affected residues are located in $\beta$-strands 3 and 4 as well as in the connecting loop (Fig. $5 \mathrm{c}$ ), in agreement with the binding site observed in the binary RfaH-CTD:S10 ${ }^{\Delta}$ complex (Fig. 5d and Supplementary Figure 6). Thus, the S10 interaction site of RfaH-CTD is accessible in the opsEC:RfaH complex, consistent with the cryo EM structure of the $\mathrm{RfaH}$ : $o p s \mathrm{EC}$ complex ${ }^{13}$ and our data that RfaH-CTD:S10 interaction is required for translation activation ${ }^{31}$.

$\mathrm{RfaH}$ is recycled upon release from the EC. The presence of $\mathrm{RfaH}-\mathrm{NTD}$ is sufficient to induce the RfaH-CTD folding into an $a$-state that is energetically unfavorable in the isolated domain $^{31,32,41}$, leading us to propose that $\mathrm{RfaH}$ transforms back into the autoinhibited state after the EC dissociates at a terminator ${ }^{32}$. Testing this hypothesis at a canonical terminator by NMR spectroscopy would be challenging because such a complex 

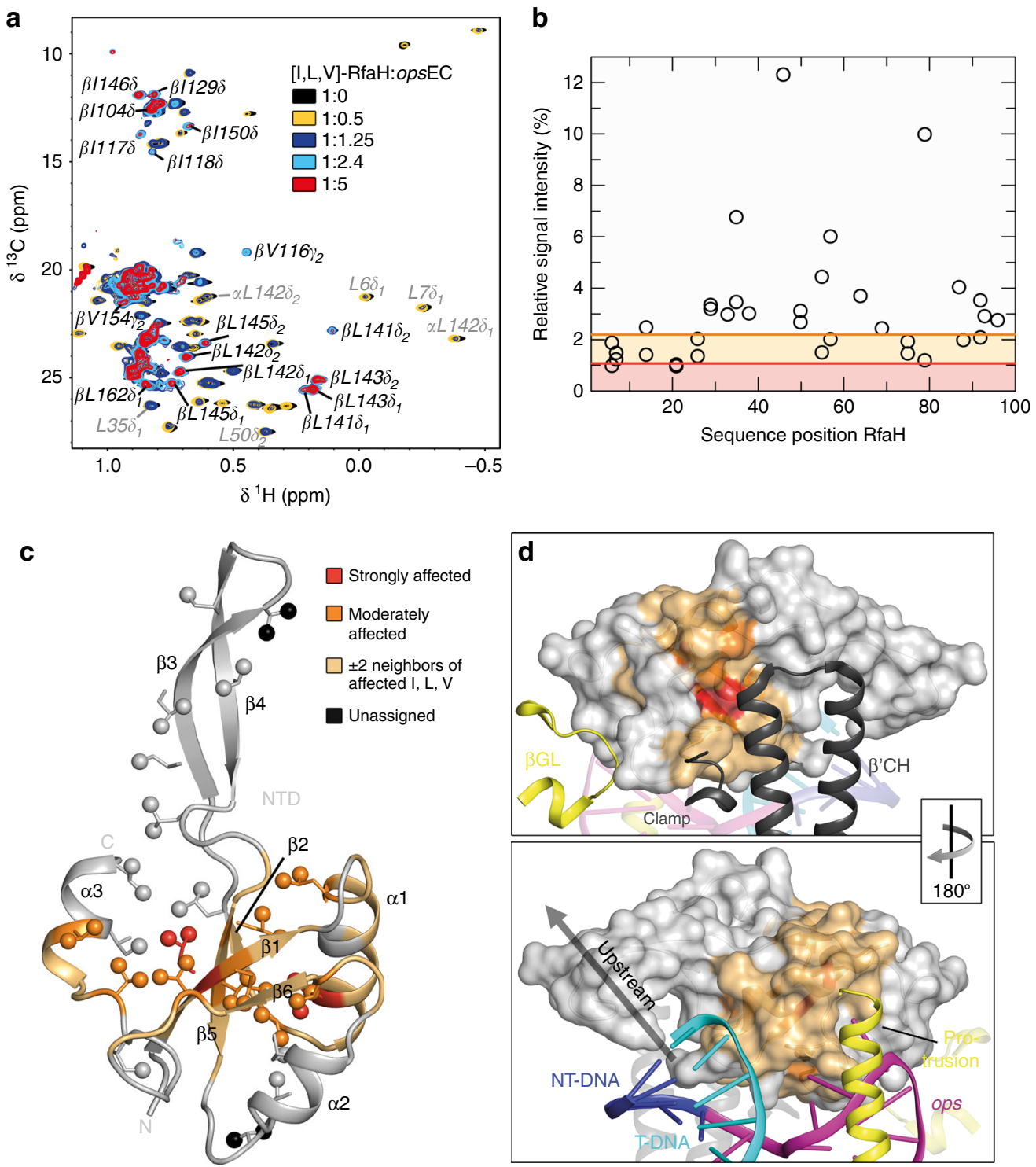

Fig. $4 \mathrm{RfaH}$ recruitment to the opsEC. a 2D $\left[{ }^{1} \mathrm{H},{ }^{13} \mathrm{C}\right]$ methyl-TROSY spectra of $[\mathrm{I}, \mathrm{L}, \mathrm{V}]-\mathrm{RfaH}$ in the absence or presence of opsEC assembled with ${ }^{2} \mathrm{H}$-RNAP (concentration of [I,L,V]-RfaH: $233 \mu \mathrm{M}(1: 0), 54 \mu \mathrm{M}(1: 0.5), 27 \mu \mathrm{M}(1: 1.25), 15 \mu \mathrm{M}(1: 2.4), 8 \mu \mathrm{M}(1: 5))$. $\alpha$ and $\beta$ indicate the all- $\alpha$ or all- $\beta$ state of the RfaHCTD. b Relative signal intensity of [I,L,V]-RfaH-NTD methyl groups with 0.5 equivalents of opsEC. Orange and red lines indicate thresholds for moderately ( $60 \%$ of average relative intensity) and strongly (30\% of average relative intensity) affected methyl groups, respectively. Source data are provided as a Source Data file. c Mapping of affected methyl groups onto RfaH-NTD structure (ribbon representation; light gray; PDB ID: '5OND'). Ile, Leu, and Val residues are in stick representation with the carbon atom of the methyl groups as sphere. Termini and secondary structure elements are labeled. The representation was graphically extended by including the two flanking residues on each side of an affected residue (beige) as established ${ }^{39}$. $\mathbf{d}$ RfaH-NTD bound to the opsEC (PDB ID: '6C6S'). RfaH-NTD is in surface representation, color code as in c, DNA and selected elements of the RNAP are in ribbon representation and labeled. The arrow indicates how the structures are rotated with respect to each other

is unstable. Instead, we induced $[\mathrm{I}, \mathrm{L}, \mathrm{V}]-\mathrm{RfaH}$ release from the opsEC by addition of a 10-fold molar excess of NusG-NTD and monitored $\mathrm{RfaH}$ displacement by recording methyl-TROSY spectra (Fig. 6a). The addition of protonated opsEC to [I,L,V]$\mathrm{RfaH}$ in a 1:1 molar ratio led to the disappearance of signals corresponding to autoinhibited $\mathrm{RfaH}$ and mainly $\beta$-barrel CTD signals were observable, confirming $\mathrm{RfaH}$ recruitment and transformation. Upon titration of [I,L,V]-RfaH:opsEC with protonated NusG-NTD, all- $\beta$ CTD signals were partially replaced by signals of autoinhibited RfaH (Fig. 6a), consistent with RfaH displacement from the opsEC followed by recycling into its autoinhibited state.

We next wanted to probe the fate of RfaH released from RNAP in a more natural pathway, upon completion of RNA synthesis.
The autoinhibited RfaH depends on wild-type (WT) ops site for recruitment and cannot act on a G8C ops template where the NTDNA hairpin is disrupted ${ }^{29}$. By contrast, the isolated RfaH-NTD can bind to the EC at any site ${ }^{30}$ and we showed that the RfaHNTD as well as RfaH variants locked in the open state due to substitutions at the NTD-CTD interface are recruited to RNAP transcribing the G8C template ${ }^{33}$. Here we used a two-step in vitro assay (Fig. 6b) to test if released $\mathrm{RfaH}$ regains its autoinhibited state, and thus dependence on ops for recruitment. In the first step, a linear DNA template containing T7A1 promoter and the ops element was immobilized on streptavidin beads via a biotin moiety. Transcription was carried out by E. coli RNAP in the presence of full-length $\mathrm{RfaH}\left(\mathrm{RfaH}^{\mathrm{FL}}\right)$ and the supernatant containing released $\mathrm{RfaH}\left(\mathrm{RfaH}^{\mathrm{SN}}\right)$ was collected. In the second 


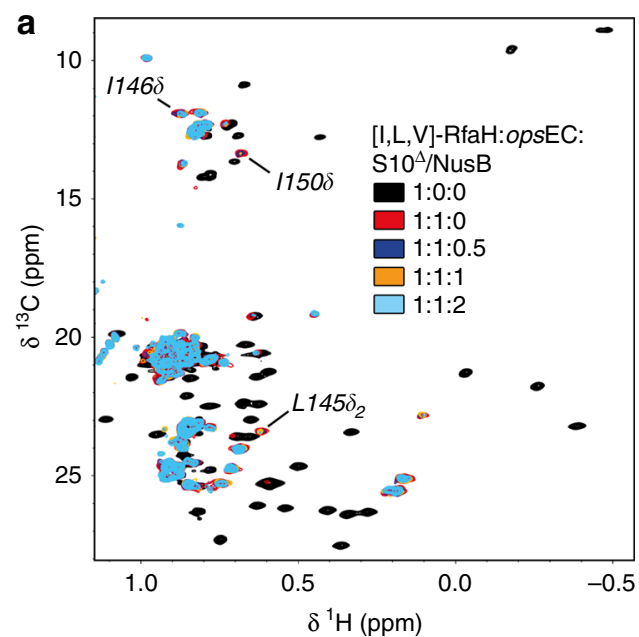

b
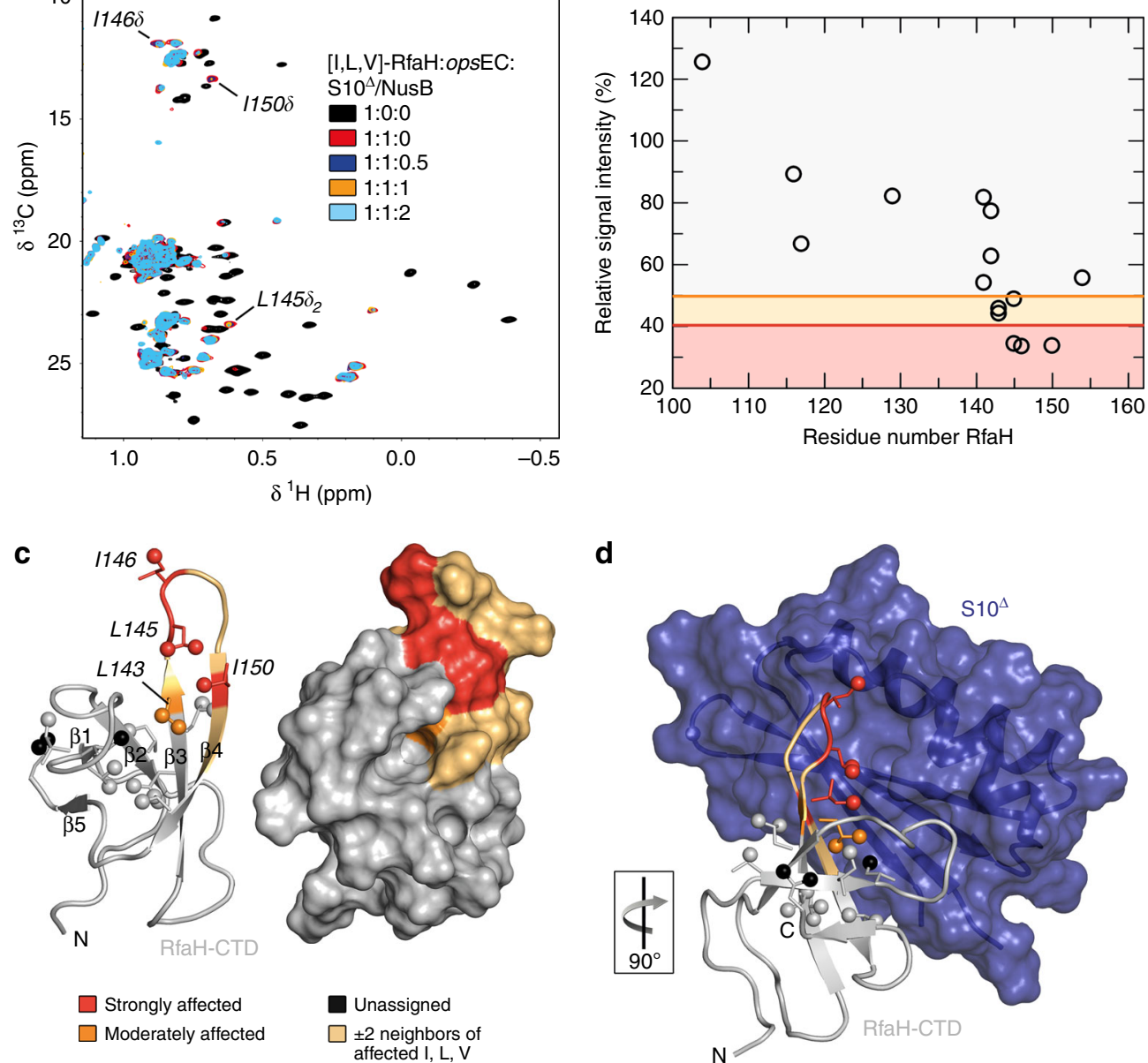

Fig. 5 Structural basis of translation activation by RfaH. a $2 \mathrm{D}\left[{ }^{1} \mathrm{H},{ }^{13} \mathrm{C}\right]$ methyl-TROSY spectra of $[\mathrm{l}, \mathrm{L}, \mathrm{V}]$-RfaH alone $(200 \mu \mathrm{M})$, in the presence of equimolar concentration of opsEC $(23 \mu \mathrm{M})$, and upon titration of RfaH:opsEC with $234 \mu \mathrm{M} \mathrm{S10}{ }^{\Delta}:$ NusB; molar ratio $[\mathrm{I}, \mathrm{L}, \mathrm{V}]-\mathrm{RfaH}: \mathrm{opsEC}_{\mathrm{S}} \mathrm{S10} \mathrm{O}^{\Delta}$ :NusB is indicated in color. Resonances with significant intensity changes are labeled. b Methyl-TROSY-derived relative intensity of [I,L,V]-RfaH methyl groups after addition of one equivalent of opsEC and two equivalents of $\mathrm{S}^{0}{ }^{\Delta}$ :NusB vs. sequence position in RfaH. Orange and red lines indicate thresholds for moderately affected ( $80 \%$ of the average relative intensity) and strongly affected ( $65 \%$ of the average relative intensity) methyl groups, respectively. Source data are provided as a Source Data file. c Mapping of affected methyl groups onto RfaH-CTD structure (PDB ID: '2LCL'). RfaH (gray) is shown in ribbon (left) and surface (right) representation, methyl groups are shown as spheres and are color-coded. $\mathbf{d}$ Model of the RfaH-CTD:S10 ${ }^{\Delta}$ complex based on the NusG-CTD:S10 ${ }^{\Delta}$ complex (PDB ID: '3D3B'). S10 ${ }^{\Delta}$ in ribbon and surface representation (blue), representation of RfaH-CTD as in $\mathbf{c}$. The orientation of RfaH-CTD relative to c is indicated

step, $\mathrm{RfaH}^{\mathrm{SN}}$ was added to halted radiolabeled ECs formed on templates with either WT or G8C ops. Following the addition of NTP substrates, the RNA products collected at different times were analyzed by gel electrophoresis (Fig. 6c) and quantified.

On the WT ops template, $\mathrm{RfaH}^{\mathrm{FL}}$ reduced RNAP pausing at U38 4-fold and delayed RNAP escape from the ops site (G39+ C40 positions) $\sim 4$-fold (Fig. 6d). RfaH-NTD and $\mathrm{RfaH}^{\mathrm{SN}}$ had very similar effects. A control in which RNAP release was prevented by a protein roadblock (RB; see Methods section) demonstrated that under these conditions all $\mathrm{RfaH}$ was bound to RNAP, as no activity was present in the supernatant. Notably, at low GTP $(5 \mu \mathrm{M})$ used in these experiments to enable manual sampling, RfaH-induced pause at G39 + C40 masks its antipausing effects downstream, and the run-off transcript yields do not increase in the presence of RfaH. As expected, RfaH-NTD stimulated productive RNA synthesis on the G8C ops template $\sim 2.5$-fold, whereas neither $\mathrm{RfaH}^{\mathrm{FL}}$ nor $\mathrm{RfaH}^{\mathrm{SN}}$ had any effect. These results suggest that $\mathrm{RfaH}$ regains the autoinhibited, opsdependent state after the EC dissociates at the end of the linear
DNA template, in support of the direct observation of reverse transformation by NMR spectroscopy (Fig. 6a).

\section{Discussion}

The results presented here support our earlier hypothesis that $\mathrm{RfaH}$ operates in a true cycle, which begins and ends with the inactive, autoinhibited state (Fig. 7). RfaH recruitment is unusually complex, with the opsEC serving as a minimal signal (Fig. 4); while $\mathrm{RfaH}$ can weakly interact with the opsDNA ${ }^{29}$ or core RNAP (Fig. 2), it binds to the opsEC with $\sim 1000$-fold higher affinity that matches its cellular concentrations ${ }^{38}$. When RNAP pauses at the ops site, the NT strand forms a hairpin, exposing the nucleotides in the loop region at the enzyme's surface, allowing sequence-specific recognition by $\mathrm{RfaH}^{13,29}$. The delay of RNAP at the ops site is thought to provide a crucial time window during which autoinhibited $\mathrm{RfaH}$ locates its few genomic targets and establishes interactions with certain RNAP elements (likely the $\beta \mathrm{GL})$ and the accessible ops nucleotides, stabilizing the NT-DNA 


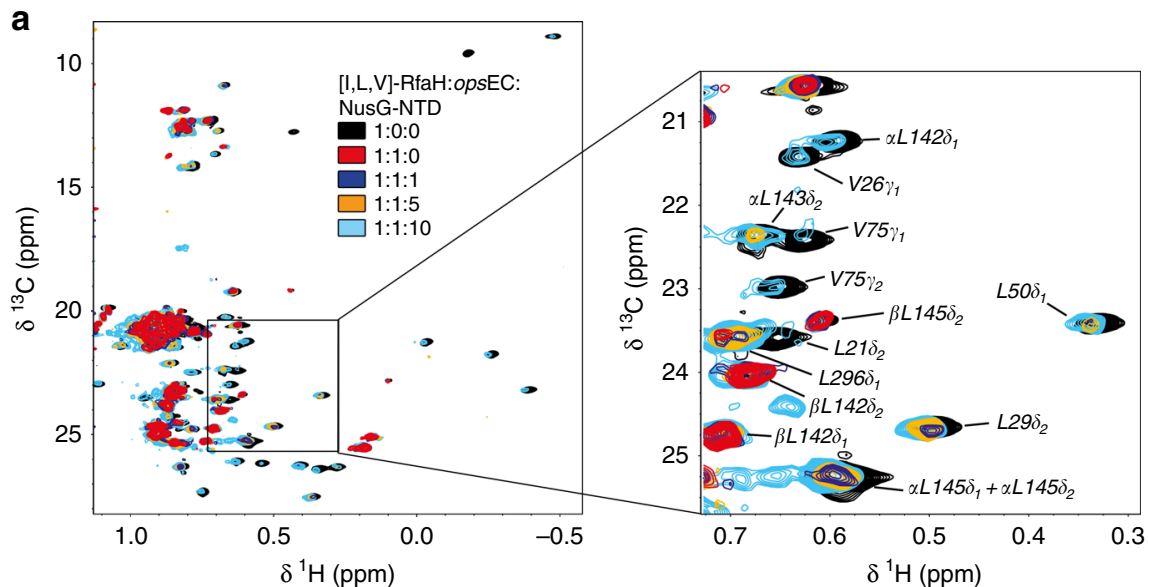

b

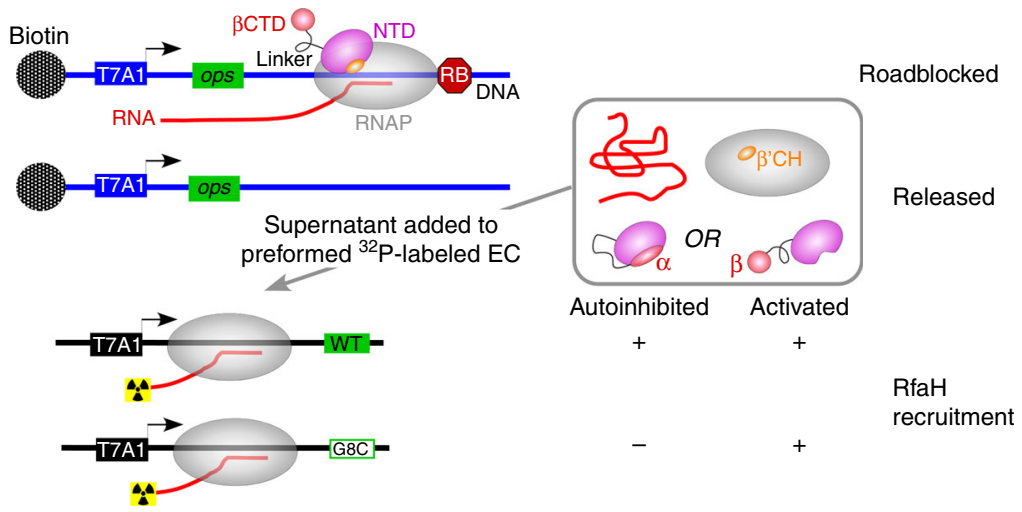

c

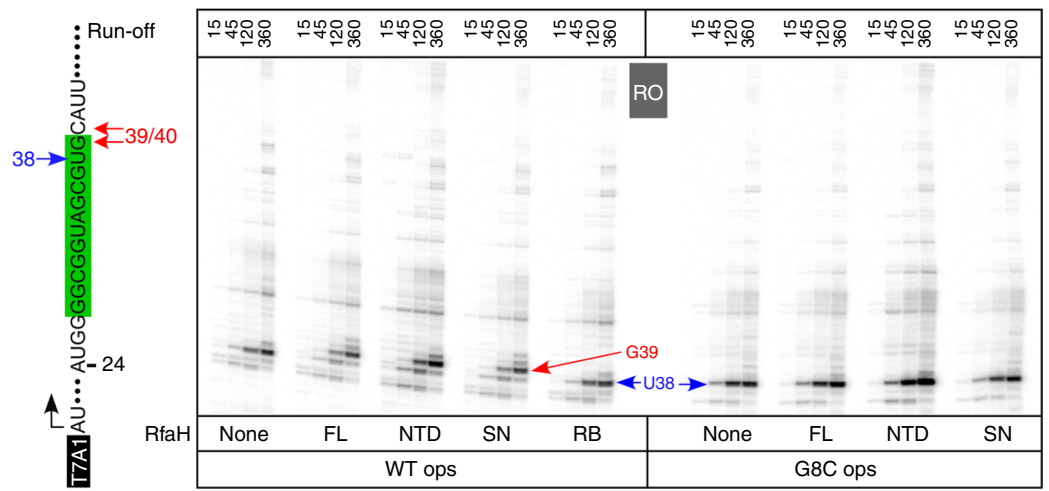

d

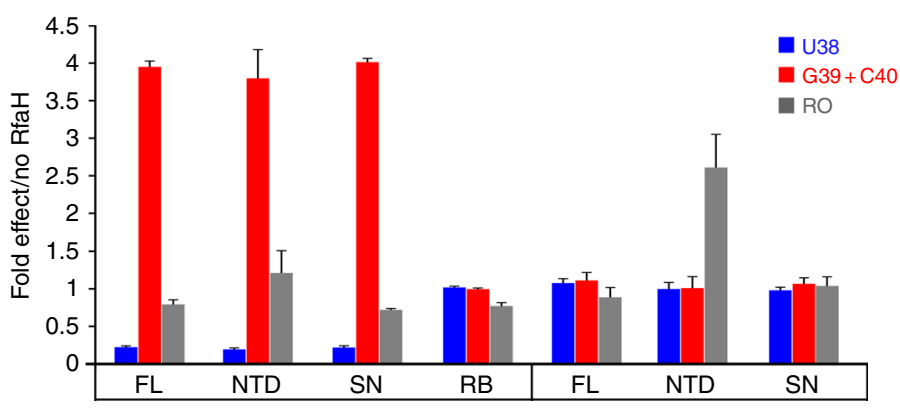

hairpin and forming a transient encounter complex. In this complex, RfaH is positioned near its final binding site on the $\beta^{\prime}$ $\mathrm{CH}$, but the high-affinity NTD: $\beta^{\prime} \mathrm{CH}$ contacts are precluded by the $\alpha$-helical CTD. As the autoinhibited state of RfaH does not exchange with an open conformation on the NMR timescale, the functional role of the encounter complex remains to be determined, although several possibilities are conceivable. (i) Contacts in the encounter complex could pre-orient $\mathrm{RfaH}$ and increase its local concentration near the $\beta^{\prime} \mathrm{CH}$, facilitating RfaH-NTD binding to the tip of the $\beta^{\prime} \mathrm{CH}$. (ii) The encounter complex could induce conformational changes that destabilize the interdomain interface and ultimately lead to transient domain opening. Although not being observable in our experiments, we cannot rule out that the binding of RfaH to opsDNA or RNAP alone may 
Fig. 6 Recycling of RfaH. a 2D $\left[{ }^{1} \mathrm{H},{ }^{13} \mathrm{C}\right]$ methyl-TROSY spectra of $[\mathrm{I}, \mathrm{L}, \mathrm{V}]-\mathrm{RfaH}$ alone $(200 \mu \mathrm{M})$, in the presence of equimolar concentration of opsEC $(23 \mu \mathrm{M}$ ), and upon titration of RfaH:opsEC with NusG-NTD (concentration of stock solutions $240 \mu \mathrm{M}$ and $486 \mu \mathrm{M}$ ); molar ratio [I,L,V]-RfaH:opsEC:NusGNTD is indicated in color. $\alpha$ and $\beta$ indicate the all- $\alpha$ or all- $\beta$ state of the RfaH-CTD. $\mathbf{b}$ Experimental set-up to follow RfaH state using in vitro transcription assay. c Determination of RfaH effect on single-round RNA synthesis. The relevant RNA region is shown on the left, with the ops element highlighted in green. Prominent pause sites (U38, G39, and C40) are indicated. Halted $\alpha^{32} \mathrm{P}$-labeled A24 ECs were chased in the presence of RfaH-NTD, RfaH ${ }^{\mathrm{FL}}$, or supernatants from roadblocked (RB) or free (SN) first-round reactions on the WT or G35C (corresponds to G8C in the ops element) template. Reactions were quenched at the indicated times (in seconds) and analyzed on 10\% denaturing acrylamide gels; a representative gel is shown. $\mathbf{d}$ The fractions of RNA species indicated were determined from 360-s time points. The ratios of RNA in the presence and in the absence of the RfaH variant indicated were determined from three independent biological replicates and are shown as mean \pm standard deviation. Source data are provided as a Source Data file

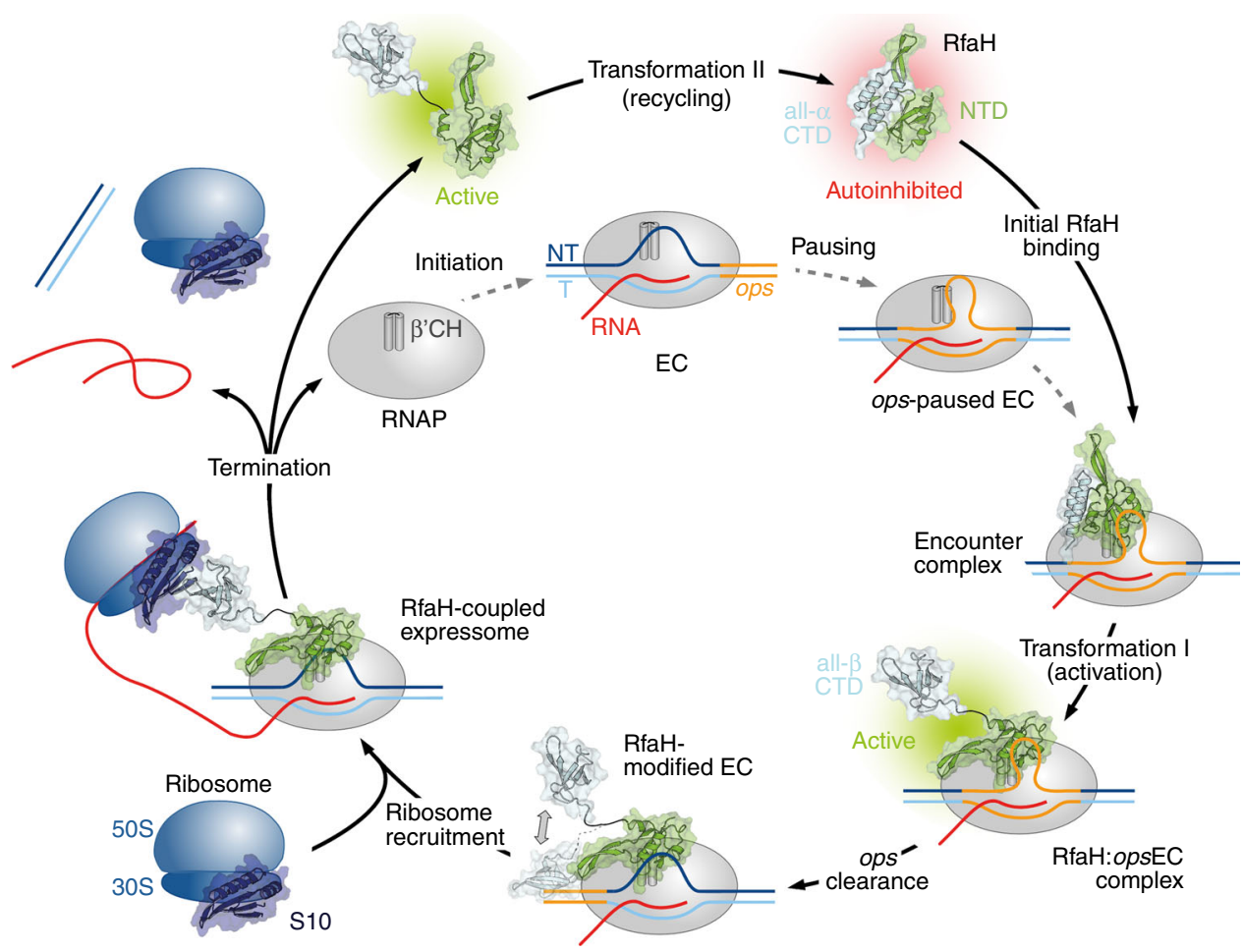

Fig. 7 Functional cycle of $\mathrm{RfaH}$. Structural transformations of the interdomain interface and the RfaH-CTD underlie reversible switching between the autoinhibited and active states of RfaH

cause such changes in the RfaH-NTD:RfaH-CTD interface. However, as only the central ops bases are exposed on the surface of the RNAP ${ }^{13}$, RfaH will inevitably interact with certain RNAP elements as soon as it establishes contacts with the ops hairpin. Thus, we hypothesize that a combination of interactions with opsDNA and RNAP provokes the weakening of the RfaH domain interface. Although we do not observe either scenario, we favor a model where elements of both mechanisms underlie RfaH activation. Upon dissociation of the $\mathrm{RfaH}$ domains the encounter complex is converted into a stable $\mathrm{RfaH}$ :EC complex. The RfaHNTD maintains interactions with the EC throughout elongation, increasing transcription processivity through stabilizing contacts with the NT-DNA and the upstream duplex DNA, as well as by blocking RNAP swiveling that occurs during pausing ${ }^{13,42}$. The released RfaH-CTD transforms and binds S10 (Fig. 5), converting $\mathrm{RfaH}$ into a potent activator of translation initiation ${ }^{31}$ and possibly coupling transcription to translation elongation, as proposed for $\mathrm{NusG}^{18}$. Finally, RfaH completes the cycle by transforming back into the autoinhibited state upon release from RNAP (Fig. 6).

Observations that all NusG homologs promote productive transcription, with the NTD being sufficient for this activity 30,43 , led us and others to focus on the NTD-dependent modification of
RNAP. Yet the regulatory diversity of NusG homologs is conferred by their CTDs, which interact with different partners to ensure coordination between RNA synthesis and posttranscriptional events. Comparison of RfaH- and NusG-CTDs reveals a combination of similar and distinct activities. Both CTDs interact similarly with $S 10^{5,31}$, suggesting that they may bridge RNAP and the lead ribosome; the available evidence is consistent with $\mathrm{RfaH}$ recruitment of ribosome $\mathrm{e}^{31}$ and with coupling by $\mathrm{NusG}^{18}$ but a systematic analysis remains to be done. In contrast, other interactions/roles are different. First, NusG-CTD binds to Rho, promoting termination at suboptimal sequences through favoring the closed, translocation-competent ring state ${ }^{6,7}$, whereas $\mathrm{RfaH}$ does not bind to Rho and inhibits Rho-dependent termination ${ }^{26}$. Second, RfaH-CTD could affect folding of the nascent RNA through transient contacts to the RNAP exit channel ${ }^{13}$, although the significance of this interaction remains to be determined. Finally, the RfaH-CTD prevents off-target recruitment and thus competition with NusG through autoinhibition mediated by transformation, maintaining the separation of the $\mathrm{RfaH}$ and NusG regulons.

Even though $\mathrm{RfaH}$ and NusG primary sequences are quite divergent ${ }^{33}$, only a few residues determine their key regulatory differences. Activation of Rho-dependent termination is 
determined by a 5-residue surface loop of NusG that, when grafted onto the RfaH-CTD, is sufficient for stimulation of Rho transition into the active conformation ${ }^{6}$. Similarly, the existence of the autoinhibited state is controlled by a few residues at the interface between the RfaH-NTD and RfaH-CTD; a single substitution of each of two key $\mathrm{RfaH}$ residues for their NusG counterparts disrupts the interface and alleviates the requirement for ops during recruitment ${ }^{33}$. In contrast, $\mathrm{RfaH}$ binding to the NT-DNA relies on readout of the primary sequence and the secondary structure of the ops hairpin by many $\mathrm{RfaH}$ residues, which are not conserved in NusG ${ }^{13,29}$.

Autoinhibition is a widespread regulatory mechanism in which intramolecular interactions between separate regions of a polypeptide negatively regulate its function, allowing temporal and spatial regulation of cellular processes by limiting activation to certain physiological conditions ${ }^{44}$. Autoinhibition modulates function of diverse proteins, from transcription factors ${ }^{45}$ to protein kinases ${ }^{46}$, and is implicated in virulence ${ }^{47}$ and disease $e^{48}$. Autoinhibition enables tight regulation, particularly when thousands of potential targets need to be distinguished, as is the case with E3 ubiquitin ligases ${ }^{49}$.

NusG cooperates with Rho to promote termination at suboptimal sites ${ }^{17}$, an essential function of NusG to silence foreign $\mathrm{DNA}^{50}$. Although being outnumbered by NusG 100:151, RfaH efficiently outcompetes NusG for binding to the $\mathrm{EC}^{32}$ and abolishes Rho-mediated termination ${ }^{26}$. To prevent interference with essential NusG, RfaH recruitment must be strictly limited to ops operons, making attainment of autoinhibition a key step in the specialization of $\mathrm{RfaH}$. Contrasting other cases of autoinhibition, autoinhibition in $\mathrm{RfaH}$ is achieved not only by the interaction of two domains, but is coupled to the transformation of a whole domain into a conformation, the all- $\alpha$ form, that does not correspond to the structure of the isolated domain ${ }^{31,32}$.

It is not known whether other specialized NusG paralogs are autoinhibited and, if so, how they are activated and whether the CTD refolds similarly. In $\mathrm{RfaH}$, transient contacts to the opsDNA hairpin and flanking RNAP regions are required to trigger domain dissociation, maybe via an encounter complex. While autoinhibited $\mathrm{RfaH}$-like proteins could use analogous contacts to the NT-DNA strand and the paused EC for activation, other mechanisms could be envisioned, such as allosteric activation; e.g., small ligands could bind to either domain to weaken their interactions.

While in E. coli NusG, the domains move independently, and no intramolecular domain interactions can be detected ${ }^{52}$, autoinhibition has been observed for Thermotoga maritima $\mathrm{NusG}^{53}$. Here, in contrast to $\mathrm{RfaH}$, autoinhibition is accomplished by the $\beta$-barrel CTD, which shields the RNAP-binding site on the NTD and most probably provides thermal stabilization ${ }^{54}$. Why does $\mathrm{RfaH}$ use an $\alpha$-helical hairpin?

To answer this question, we generated a model of $\mathrm{RfaH}$ where the all- $\beta$ CTD interacts with the NTD as in TmNusG (RfaH ${ }^{\beta C T D}$; Supplementary Figure 7). The linker is sufficiently long and $\mathrm{RfaH}^{\beta C T D}$ can be easily integrated into a closed conformation without steric problems. Analysis of the domain interactions reveals that in $\mathrm{RfaH}$ the binding surface is larger than in $\mathrm{RfaH}^{\beta C T D}\left(\sim 900 \AA^{2}\right.$ vs. $\left.\sim 700 \AA^{2}\right)$, resulting in a significantly more negative solvation energy $(\sim-14 \mathrm{kcal} / \mathrm{mol}$ vs. $-8 \mathrm{kcal} / \mathrm{mol})$. The stronger domain interaction in $\mathrm{RfaH}$ may be required to prevent an equilibrium between open and closed state, consistent with our present results, and thus uncontrolled activation.

We speculate that a need to tightly control the off-target recruitment necessitates the transformation-coupled autoinhibition in $\mathrm{RfaH}$, especially as the use of the all-a CTD state imparts dual autoinhibition-the closed state lacks the binding sites for both RNAP and the ribosome, potentially further minimizing deleterious effects of spurious $\mathrm{RfaH}$ activation. Studies of structures and recruitment of NusG paralogs from other species will reveal their underlying specificity mechanisms.

The thermodynamic hypothesis states that, under physiological conditions, a protein adopts the three-dimensional structure which corresponds to the state of the lowest Gibbs free energy for the whole system. This so-called physiological state is solely determined by the totality of interactions and thus the amino acid sequence ${ }^{55}$. Most proteins exist as an ensemble of closely related equilibrium structures in their energetically stable state and thus follow this one-sequence, one-fold paradigm. However, more and more chameleonic/metamorphic proteins ${ }^{56}$ that defy this paradigm are found [see ref. ${ }^{57}$ for a recent review]. In these metamorphic proteins, more than one distinct structural form is energetically favored.

In most cases, the metamorphic regions are small (5-14 residues) and metamorphosis involves either transitions between unstructured to structured states or conformational switching $(\alpha \leftrightarrow \beta)$. Transitions can be triggered by simple external cues (e.g., $\mathrm{pH}$, temperature, salt concentration; refs. ${ }^{58,59}$ ) may be driven by evolutionary pressure as observed in the Cro family of bacteriophage transcription factors ${ }^{60}$, or may underlie biological activity, such as regulation of chemotaxis by lymphotactin ${ }^{61}$, pore formation by lytic toxins ${ }^{62}$, regulation of the circadian clock by $\mathrm{KaiB}^{63}$, or photoreactivation in cytochromes ${ }^{64}$. As in classical metamorphoses, the observed changes are usually unidirectional, although reversible refolding has been reported ${ }^{61,63,65}$.

What sets $\mathrm{RfaH}$ apart from other metamorphic proteins are (i) the scale of the reversible transformation, in which the entire 50residue domain refolds, (ii) distinct and essential biological functions of both alternative folds, and (iii) the fact that this dramatic behavior occurs in a member of the only universally conserved family of transcription factors. The fold of the CTD is solely determined by the presence or absence of the $\mathrm{NTD}^{32}$, i.e., the information which fold to adopt is encoded in $\mathrm{RfaH}$ itself. A major determinant of the NTD:CTD interaction is the salt bridge E48:R138 as its elimination leads to a coexistence of the autoinhibited state and the open form with the CTD in the $\beta$-barrel conformation $^{31}$, turning $\mathrm{RfaH}$ into a NusG-like general transcription factor as the dependence on ops is abolished ${ }^{31}$. Arguments (i) and (ii) prompted us to name $\mathrm{RfaH}$ a transformer protein $^{34}$. An $\alpha \rightarrow \beta$ switch of a whole protein/domain is only known for amyloidogenic proteins, such as prions ${ }^{66}$, but the two states cannot coexist, the transition is irreversible, and the resulting $\beta$-aggregates are pathogenic.

In conclusion, metamorphosis is an increasingly recognized regulatory tool in nature, but the functional and conformational plasticity coupled with autoinhibition of $\mathrm{RfaH}$ sets new standards for regulation and suggests that similar principles are exploited by many transformer proteins awaiting discovery.

\section{Methods}

Cloning. The gene encoding $\mathrm{RfaH}$ was amplified from pIA238 using primers Fw_rfaH_pET19bmod and Rv_rfaH_pET19bmod (Supplementary Table 2) and cloned into pET19bmod, a variant of pET19b, via NdeI and BamHI restriction sites. The recombinant target protein carries a hexahistidine $\left(\mathrm{His}_{6}\right)$ tag followed by a tobacco etch virus (TEV) protease cleavage site at its N-terminus.

Gene expression and protein purification. All E. coli strains used in gene expression were derivatives of E. coli $\mathrm{B}$ and grown at temperatures between 16 and $37^{\circ} \mathrm{C}$. Antibiotics were added to the medium as follows: ampicillin $100 \mu \mathrm{g} / \mathrm{ml}$, carbenicillin $100 \mu \mathrm{g} / \mathrm{ml}$, kanamycin $50 \mu \mathrm{g} / \mathrm{ml}$, and chloramphenicol $34 \mu \mathrm{g} / \mathrm{ml}$. The source organisms for all proteins used in this work are derivatives of $E$. coli K. All expression plasmids are listed in Supplementary Table 3.

$\mathrm{RfaH}$ for NMR studies was produced as described in ref. ${ }^{31}$. In brief, E. coli BL21 ( $\lambda$ DE3) cells (Novagen, Madison, WI, USA) harboring plasmid pET19bmod_RfaH were grown in lysogeny broth (LB) medium supplemented with kanamycin to an optical density at $600 \mathrm{~nm}\left(O D_{600}\right)$ of 0.6 at $37^{\circ} \mathrm{C}$. The temperature was lowered to 
$20^{\circ} \mathrm{C}$ and gene expression was induced after $30 \mathrm{~min}$ by addition of $0.2 \mathrm{mM}$ isopropyl-1-thio- $\beta$-D-galactopyranoside (IPTG). Cells were harvested after overnight incubation by centrifugation $(6000 \times g)$, resuspended in buffer $\mathrm{A}^{\mathrm{RfaH}}$ (50 mM tris(hydroxymethyl)aminomethane (Tris)/ $\mathrm{HCl}(\mathrm{pH} 7.5), 300 \mathrm{mM} \mathrm{NaCl}$, $5 \%$ (v/v) glycerol, $1 \mathrm{mM}$ dithiothreitol (DTT)) supplemented with $10 \mathrm{mM}$ imidazole, DNase I (AppliChem GmbH, Darmstadt, Germany), and 1/2 protease inhibitor tablet (cOmplete, EDTA-free, Roche Diagnostics, Mannheim, Germany) and lysed using a microfluidizer. The lysate was cleared by centrifugation and the soluble fraction was then applied to a HisTrap column (column volume 1-5 ml, GE Healthcare, Munich, Germany) that was subsequently washed with buffer $\mathrm{A}^{\mathrm{RfaH}}$ supplemented with $10 \mathrm{mM}$ imidazole. A step gradient from $100 \mathrm{mM}$ to $1 \mathrm{M}$ imidazole in buffer $\mathrm{A}^{\mathrm{RfaH}}$ was used for elution. RfaH-containing fractions were combined and dialyzed against buffer $\mathrm{B}^{\mathrm{RfaH}}(50 \mathrm{mM}$ Tris/ $\mathrm{HCl}(\mathrm{pH} 7.5), 150 \mathrm{mM}$ $\mathrm{NaCl}, 5 \%(\mathrm{v} / \mathrm{v})$ glycerol, $1 \mathrm{mM} \mathrm{DTT})$. After $2 \mathrm{~h}$ TEV protease was added and cleavage was carried out overnight at $4{ }^{\circ} \mathrm{C}$. The solution was then again applied on a $5 \mathrm{ml}$ HisTrap column (GE Healthcare, Munich, Germany). The target protein was collected in the flow-through, concentrated by ultrafiltration, flash-frozen in liquid nitrogen, and stored at $-80^{\circ} \mathrm{C}$.

$\mathrm{RfaH}$ for transcription assays was produced similarly, except that plasmid pIA238 was used for the expression, resulting in E. coli $\mathrm{RfaH}$ with $\mathrm{N}$-terminal $\mathrm{His}_{6}{ }^{-}$ tag followed by a thrombin cleavage site. Thus, cleavage was carried out during overnight dialysis at room temperature in the presence of thrombin instead of TEV protease.

The production of RfaH-CTD was according to ref. ${ }^{31}$ and the conditions were similar to the ones used for full-length RfaH. For expression E. coli BL21 (DE3) cells containing pETGB1a_EcrfaH-CTD(101-162) were used. The plasmid codes for $E$. coli $\mathrm{RfaH}$-CTD with $\mathrm{N}$-terminal $\mathrm{His}_{6}$-Gb1 tag followed by a TEV protease cleavage site. For purification a $5 \mathrm{ml} \mathrm{Ni}^{2+}$-HiTrap column (GE Healthcare, Munich, Germany) was used and buffer $\mathrm{A}^{\mathrm{RfaH}-\mathrm{CTD}}$ consisted of $50 \mathrm{mM}$ Tris/ $\mathrm{HCl}(\mathrm{pH} 7.5)$, $150 \mathrm{mM} \mathrm{NaCl}$. The pure target protein was finally dialyzed against $25 \mathrm{mM} \mathrm{4-(2-}$ hydroxyethyl)-1-piperazineethanesulfonic acid (HEPES; pH 7.5), $100 \mathrm{mM} \mathrm{NaCl}$, concentrated by ultrafiltration, flash-frozen in liquid nitrogen, and stored at $-80^{\circ} \mathrm{C}$.

For the production of NusG-NTD ${ }^{52}$ E. coli BL21 ( $\lambda$ DE3) cells harboring plasmid pET11a_EcNusG-NTD(1-124), which encodes E. coli NusG 1-124, were grown in ampicillin-containing $\mathrm{LB}$ medium to an $O D_{600}$ of 0.8 at $37^{\circ} \mathrm{C}$. Overexpression was induced by addition of $1 \mathrm{mM}$ IPTG. After $4 \mathrm{~h}$ cells were harvested by centrifugation $(6000 \times g)$, resuspended in buffer $\mathrm{A}^{\text {NusG-NTD }}(50 \mathrm{mM}$ Tris/HCl (pH 7.5), $150 \mathrm{mM} \mathrm{NaCl}$ ) supplemented with DNase I (AppliChem $\mathrm{GmbH}$, Darmstadt, Germany) and 1/4 protease inhibitor tablet (cOmplete, EDTAfree, Roche Diagnostics, Mannheim, Germany), and lysed with a microfluidizer. After centrifugation, nucleic acids were precipitated by addition of streptomycine sulfate $(1 \%(\mathrm{w} / \mathrm{v}))$. Upon centrifugation $\left(\mathrm{NH}_{4}\right)_{2} \mathrm{SO}_{4}$ was added to the supernatant to a concentration of $50 \%(\mathrm{w} / \mathrm{v})$, precipitating NusG-NTD. The precipitate was pelleted by centrifugation and dissolved in buffer $\mathrm{B}^{\mathrm{NusG}-\mathrm{NTD}}(10 \mathrm{mM}$ Tris/HCl $(\mathrm{pH}$ 7.5)). The solution was dialyzed against buffer $\mathrm{B}^{\mathrm{NusG}-\mathrm{NTD}}$ before being applied to a $5 \mathrm{ml}$ HeparinFF column (GE Healthcare, Munich, Germany). The column was washed with buffer $\mathrm{B}^{\text {NusG-NTD }}$ and the target protein was eluted by a $\mathrm{NaCl}$ step gradient from $50 \mathrm{mM}$ to $1 \mathrm{M}$ in buffer $\mathrm{B}^{\text {NusG-NTD }}$. NusG-NTD containing fractions were combined, concentrated by ultrafiltration, and applied to a HiLoad S75 size exclusion column (GE Healthcare, Munich, Germany) equilibrated with buffer $\mathrm{C}^{\text {NusG-NTD }}$ (25 mM HEPES (pH 7.5), $100 \mathrm{mM} \mathrm{NaCl}$ ). NusG-NTD containing fractions were combined and the solution was concentrated by ultrafiltration and flash-frozen in liquid nitrogen before being stored at $-80^{\circ} \mathrm{C}$.

The production of $\mathrm{S}^{\Delta} 0^{\Delta}$ :NusB was based on ref. ${ }^{67}$. Briefly, E. coli BL21 ( $\lambda$ DE3) cells harboring the plasmids for either $\mathrm{S}_{10}{ }^{\Delta}$ (pGEX-6P_ecoNusE ${ }^{\Delta}$; encodes $E$. coli $\mathrm{S} 0^{\Delta}$ with N-terminal glutathione S-transferase (GST)-tag followed by PreScission protease cleavage site) or NusB (pET29b ecoNusB; encodes E. coli NusB), were grown in LB medium containing ampicillin or kanamycin, respectively, at $37^{\circ} \mathrm{C}$ to an $\mathrm{OD}_{600}$ of 0.5 . The temperature was lowered to $20^{\circ} \mathrm{C}$ and gene expression was induced after $30 \mathrm{~min}$ by addition of $0.5 \mathrm{mM}$ IPTG. After overnight incubation cells were harvested by centrifugation $(6000 \times g)$. Cell pellets of $\mathrm{S}^{\Delta} 0^{\Delta}$ and NusBcontaining cells, obtained from the same culture volume, were resuspended in buffer $\mathrm{A}^{\mathrm{S} 10 \Delta \mathrm{NusB}}$ (50 mM Tris/HCl (pH 7.5), $150 \mathrm{mM} \mathrm{NaCl}, 1 \mathrm{mM}$ DTT) and combined. Cells were subsequently lysed using a microfluidizer and the lysate was stirred for $30 \mathrm{~min}$ at $4^{\circ} \mathrm{C}$ to ensure formation of the $S 10^{\Delta}$ :NusE complex. The extract was then cleared by centrifugation and applied to four coupled $5 \mathrm{ml}$ GSTrap FF columns (GE Healthcare, Munich, Germany) equilibrated with buffer $\mathrm{A}^{\mathrm{S} 10 \Delta}$ NusB . After washing with buffer $\mathrm{A}^{\mathrm{S} 10 \Delta \text { :NusB }}$ the complex was eluted with buffer $\mathrm{A}^{\mathrm{S} 10 \Delta \mathrm{N} \text { NusB }}$ containing $15 \mathrm{mM}$ reduced glutathione. The $\mathrm{S}_{10}{ }^{\Delta}$ :NusB solution was supplemented with PreScission protease and dialyzed against buffer $\mathrm{B}^{\mathrm{S} 10 \Delta \text { :NusB }}$ (50 mM Tris/HCl (pH 7.5), $1 \mathrm{mM} \mathrm{DTT)} \mathrm{overnight.} \mathrm{The} \mathrm{protein} \mathrm{solution} \mathrm{was}$ applied to two $5 \mathrm{ml}$ HiTrap Q XL columns (GE Healthcare, Munich, Germany) coupled to two HiTrap SP XL columns (GE Healthcare, Munich, Germany). Upon washing with buffer $B^{S 10 \Delta: N u s B}$ the HiTrap SP XL columns were disconnected and $\mathrm{S}_{10}{ }^{\Delta}$ :NusB were eluted with buffer $\mathrm{B}^{\mathrm{S} 10 \Delta}$ :NusB containing $1 \mathrm{M} \mathrm{NaCl}$. The solution was dialyzed against $25 \mathrm{mM}$ HEPES ( $\mathrm{pH} 7.5$ ), $100 \mathrm{mM} \mathrm{NaCl}$, concentrated by ultrafiltration, before being flash-frozen in liquid nitrogen, and stored at $-80^{\circ} \mathrm{C}$.

RNAP for in vitro transcription assays was produced according to ref. ${ }^{68}$. E. coli BL21 ( $\lambda$ DE3) cells harboring pVS10 (encoding E. coli RNAP subunits $\alpha, \beta, \beta^{\prime}$ with $\mathrm{C}$-terminal $\mathrm{His}_{6}$ tag, and $\omega$ ) were grown at $37^{\circ} \mathrm{C}$ in carbenicillin-containing $\mathrm{LB}$ medium to an $\mathrm{OD}_{600}$ of 0.75 before overexpression was induced by $1 \mathrm{mM}$ IPTG for
$3 \mathrm{~h}$. Cells were harvested by centrifugation $(6000 \times g)$ and resuspended in buffer $\mathrm{A}^{\mathrm{RNAP1}}$ (50 mM Tris/HCl (pH 6.9), $500 \mathrm{mM} \mathrm{NaCl}, 5 \%$ (v/v) glycerol) supplemented with one protease inhibitors cocktail (Roche Applied Science) and $1 \mathrm{mg} / \mathrm{ml}$ lysozyme. Cell lysis was carried out by sonication and the cleared extract was supplemented with $20 \mathrm{mM}$ imidazole before being loaded onto a His GraviTrap column (GE Healthcare Life Science). The column was washed with buffer $\mathrm{A}^{\mathrm{RNAP1}}$ containing $20 \mathrm{mM}$ imidazole and RNAP was eluted with buffer $\mathrm{A}^{\mathrm{RNAP} 1}$ containing $250 \mathrm{mM}$ imidazole. The protein solution was dialyzed against buffer $\mathrm{B}^{\mathrm{RNAP1}}$ (50 mM Tris/HCl (pH 6.9), 5\% (v/v) glycerol, $0.5 \mathrm{mM}$ EDTA, $1 \mathrm{mM}$ DTT) supplemented with $75 \mathrm{mM} \mathrm{NaCl}$ and was then applied to a HiPrep Heparin FF column (GE Healthcare Life Science) to remove nucleic acids. The column was washed with buffer $\mathrm{B}^{\mathrm{RNAPl}}$ containing $75 \mathrm{mM} \mathrm{NaCl}$ and RNAP was eluted with a constant $\mathrm{NaCl}$ gradient from $75 \mathrm{mM}$ to $1.5 \mathrm{M}$ in buffer $\mathrm{B}^{\mathrm{RNAP1}}$. Target protein containing fractions were dialyzed against buffer $\mathrm{B}^{\mathrm{RNAP} 1}$ containing $75 \mathrm{mM} \mathrm{NaCl}$ and applied on a MonoQ column (GE Healthcare Life Science). Washing and elution were analogous to the Heparin affinity chromatography step. RNAPcontaining fractions were combined, dialyzed against $10 \mathrm{mM}$ Tris/ $\mathrm{HCl}(\mathrm{pH} 7.5)$, $100 \mathrm{mM} \mathrm{NaCl}, 50 \%$ (v/v) glycerol, $0.1 \mathrm{mM}$ EDTA, $0.1 \mathrm{mM} \mathrm{DTT}$, and stored at $-20{ }^{\circ} \mathrm{C}$.

The production of RNAP for NMR studies was based on ref. ${ }^{69}$. Expression was carried out in E. coli BL21 ( $\lambda$ DE3) containing plasmid pVS10. Cells were grown in $\mathrm{LB}$ medium supplemented with ampicillin to an $\mathrm{OD}_{600}$ of 0.7 . The temperature was lowered to $16^{\circ} \mathrm{C}$ and overexpression was induced at $\mathrm{OD}_{600}=0.8$ with $0.5 \mathrm{mM}$ IPTG. After overnight growth cells were harvested by centrifugation. The pellet was resuspended in buffer $\mathrm{A}^{\mathrm{RNAP} 2}(50 \mathrm{mM}$ Tris/ $\mathrm{HCl}(\mathrm{pH} 6.9), 500 \mathrm{mM} \mathrm{NaCl}, 5 \%$ (v/v) glycerol, $1 \mathrm{mM} \beta$-mercaptoethanol ( $\beta$-ME)) containing $10 \mathrm{mM}$ imidazole, DNase I (AppliChem GmbH, Darmstadt, Germany), and 1/2 protease inhibitor tablet (cOmplete, EDTA-free, Roche Diagnostics, Mannheim, Germany). Cells were lysed using a microfluidizer and the lysate was cleared by centrifugation. The supernatant was applied to a $40 \mathrm{ml} \mathrm{Ni}^{2+}$-Chelating Sepharose column (GE Healthcare, Munich, Germany). After washing with buffer $A^{\text {RNAP2 }}$ containing $10 \mathrm{mM}$ imidazole RNAP was eluted using an imidazole gradient from $90 \mathrm{mM}$ to $1 \mathrm{M}$ imidazole in buffer $\mathrm{A}^{\mathrm{RNAP} 2}$. RNAP-containing fractions were dialyzed against buffer B $\mathrm{B}^{\mathrm{RNAP} 2}(50 \mathrm{mM}$ Tris/HCl $(\mathrm{pH}$ 6.9), 5\% (v/v) glycerol, $0.5 \mathrm{mM}$ EDTA, $1 \mathrm{mM}$ $\beta-\mathrm{ME}$ ) containing $100 \mathrm{mM} \mathrm{NaCl}$ and then applied to two coupled $5 \mathrm{ml}$ Heparin FF columns (GE Healthcare, Munich, Germany). The columns were washed with buffer B $\mathrm{B}^{\mathrm{RNAP} 2}$ (containing $100 \mathrm{mM} \mathrm{NaCl}$ ) and RNAP was eluted with a constant gradient from $100 \mathrm{mM}$ to $1 \mathrm{M} \mathrm{NaCl}$ in buffer $\mathrm{B}^{\mathrm{RNAP} 2}$. The fractions containing core RNAP were dialyzed against buffer $C^{\mathrm{RNAP} 2}(50 \mathrm{mM}$ Tris/HCl (pH 6.9), $150 \mathrm{mM} \mathrm{NaCl}, 5 \%$ (v/v) glycerol, $0.5 \mathrm{mM}$ EDTA, $1 \mathrm{mM} \beta$-ME) and subsequently concentrated by ultrafiltration. The concentrate was applied to a HiLoad S200 size exclusion column (GE Healthare, Munich, Germany) equilibrated with buffer $\mathrm{C}^{\mathrm{RNAP} 2}$ to remove inactive RNAP aggregates. Fractions containing pure, active enzyme were concentrated by ultrafiltration, glycerol was added to a final concentration of $50 \%(\mathrm{v} / \mathrm{v})$ and the protein solution was stored at $-20^{\circ} \mathrm{C}$.

Protein purity was checked by SDS-PAGE, the absence of nucleic acids was checked by recording UV/Vis spectra on a Nanodrop ND-1000 spectrometer (PEQLAB, Erlangen, Germany). Concentrations were determined by measuring the absorbance at $280 \mathrm{~nm}\left(A_{280}\right)$ in a $10 \mathrm{~mm}$ quartz cuvette (Hellma, Müllheim, Germany) on a Biospectrometer basic (Eppendorf, Hamburg, Germany).

Isotopic labeling. ${ }^{15} \mathrm{~N}$ - and ${ }^{15} \mathrm{~N} /{ }^{13} \mathrm{C}$-labeled proteins were produced by growing $E$. coli cells in $\mathrm{M} 9$ medium ${ }^{70,71}$ containing $\left({ }^{15} \mathrm{NH}_{4}\right)_{2} \mathrm{SO}_{4}$ and ${ }^{13} \mathrm{C}-\mathrm{D}$-glucose. For the production of perdeuterated proteins, cells were grown in $\mathrm{M} 9$ medium $^{70,71}$ prepared with increasing amounts of $\mathrm{D}_{2} \mathrm{O}\left(25 \%(\mathrm{v} / \mathrm{v}), 50 \%(\mathrm{v} / \mathrm{v}), 99.9 \%(\mathrm{v} / \mathrm{v}) \mathrm{D}_{2} \mathrm{O}\right.$; Eurisotop, Saint-Aubin, France) with $\mathrm{d}_{7}$-glucose as carbon source. The site-specific $\left[{ }^{1} \mathrm{H},{ }^{13} \mathrm{C}\right]$-labeling of Ile, Leu, and Val methyl groups in perdeuterated proteins was performed according to published protocols ${ }^{72}$, i.e., expression was carried out as described for the production of perdeuterated proteins, but the medium contained $\mathrm{d}_{7}$-glucose as carbon source and $60 \mathrm{mg} / \mathrm{l} 2$-keto-3- $\mathrm{d}_{3}-4-{ }^{13} \mathrm{C}$-butyrate and $100 \mathrm{mg} / \mathrm{l}$ 2-keto-3-methyl- $\mathrm{d}_{3}-3-\mathrm{d}_{1}-4-{ }^{13} \mathrm{C}$-butyrate (both from Eurisotop, St. Aubin Cedex, France) were added $1 \mathrm{~h}$ prior to induction. Expression and purification were as described for the production of unlabeled proteins.

NMR spectroscopy. NMR experiments were performed on Bruker Avance 700 $\mathrm{MHz}$, Bruker Ascend Aeon $900 \mathrm{MHz}$, and Bruker Ascend Aeon $1000 \mathrm{MHz}$ spectrometers. All spectrometers were equipped with cryogenically cooled, inverse triple resonance probes. Processing of NMR data was carried out using in-house routines. 2D/3D spectra were visualized and analyzed by NMRViewJ (One Moon Scientific, Inc., Westfield, NJ, USA), 1D spectra by MatLab (The MathWorks, Inc., Version 7.1.0.183). Measurements were conducted at $15^{\circ} \mathrm{C}$. The initial sample volume was $500 \mu \mathrm{l}$, if not stated otherwise.

The resonance assignments for the backbone amide groups of $\mathrm{RfaH}$ and for the methyl groups of RfaH-CTD were taken from a previous study ${ }^{31}$. For resonance assignment of the RfaH methyl groups $\left[{ }^{13} \mathrm{C},{ }^{15} \mathrm{~N}\right]-\mathrm{RfaH}$ in $25 \mathrm{mM}$ HEPES ( $\mathrm{pH}$ 7.5), $50 \mathrm{mM} \mathrm{NaCl}, 5 \%$ (v/v) glycerol, $1 \mathrm{mM} \mathrm{DTT}, 10 \% \mathrm{D}_{2} \mathrm{O}$ and [I,L,V]-RfaH in $50 \mathrm{mM} \mathrm{Na} \mathrm{HPO}_{4} / \mathrm{NaH}_{2} \mathrm{PO}_{4}$ (pH 7.5), $50 \mathrm{mM} \mathrm{KCl,} 0.3 \mathrm{mM}$ EDTA, 99.9\% $\mathrm{D}_{2} \mathrm{O}$ were used. The assignment was based on standard double and triple resonance experiments on $\left[{ }^{13} \mathrm{C},{ }^{15} \mathrm{~N}\right]-\mathrm{RfaH}$ with $(\mathrm{H}) \mathrm{CCH}$-total correlation spectroscopy (TOCSY) and $\mathrm{H}(\mathrm{C}) \mathrm{CH}-\mathrm{TOCSY}$ spectra allowing the non-sequence-specific 
identification of peaks belonging to the two methyl groups within individual Leu or Val side chains. Additionally, 3D CCH- and $\mathrm{HCH}$-nuclear Overhauser effect spectroscopy (NOESY) spectra (mixing times: 250 and $200 \mathrm{~ms}$, respectively) were obtained from $[\mathrm{I}, \mathrm{L}, \mathrm{V}]-\mathrm{RfaH}$. Combining the NOESY patterns with structural information from the crystal structure of the RfaH:ops 9 complex (protein data bank (PDB) ID: '5OND'), and the identification of associated methyl groups finally allowed for the assignment of most non-overlapping resonances.

For interaction studies involving RNAP all components were in $50 \mathrm{mM}$ $\mathrm{Na}_{2} \mathrm{HPO}_{4} / \mathrm{NaH}_{2} \mathrm{PO}_{4}$ (pH 7.5), $50 \mathrm{mM} \mathrm{KCl}, 0.3 \mathrm{mM}$ EDTA, 99.9\% (v/v) $\mathrm{D}_{2} \mathrm{O}$.

Interaction studies with chemical shifts changes in the fast regime on the chemical-shift timescale were analyzed by calculating the normalized chemicalshift perturbation $\left(\Delta \delta_{\text {norm }}\right)$ according to Eq. (1) for $\left[{ }^{1} \mathrm{H},{ }^{13} \mathrm{C}\right]$ correlation spectra.

$$
\Delta \delta_{\text {norm }}=\sqrt{\left(\Delta \delta^{1} \mathrm{H}\right)^{2}+\left[0.25\left(\Delta \delta^{13} \mathrm{C}\right)\right]^{2}}
$$

where $\Delta \delta$ is the resonance frequency difference in ppm.

To analyze the signal intensity quantitatively in both $1 \mathrm{D}$ and $2 \mathrm{D}$ experiments, the intensity was normalized by the concentration of the labeled protein, the number of scans, the receiver gain, and the length of the $90^{\circ}$ proton pulse. The ratio of remaining signal intensities and signal intensities in the spectrum of the free, labeled protein was calculated for each titration step, resulting in relative signal intensities. The determination of the interaction surface of ops EC and S10 on RfaH was carried out as described in ref. ${ }^{39}$. In brief, the mean value of all relative signal intensities in each titration step was determined and experiment-specific thresholds of the mean value were defined. Residues with relative signal intensities below these thresholds were classified as either strongly or moderately affected and Leu and Val residues were considered as affected if at least one of the two signals showed a significant decrease in intensity. Only unambiguously assigned signals were used in the analysis. Affected residues were mapped on the threedimensional structure of $\mathrm{RfaH} / \mathrm{RfaH}-\mathrm{CTD}$ and binding surfaces were graphically extended by (i) highlighting the complete amino acids instead of only the methyl group and (ii) highlighting the two amino acids on either side of an affected Ile, Leu, or Val residue unless they were unaffected/unassigned Ile, Leu, Val residues.

Translational diffusion coefficients $(D)$ were determined using a stimulated echo (STE) experiment combined with a $1 \mathrm{D}\left[{ }^{1} \mathrm{H},{ }^{13} \mathrm{C}\right]-\mathrm{HMQC}$ for selecting ${ }^{13} \mathrm{C}$ bound protons using an $[\mathrm{I}, \mathrm{L}, \mathrm{V}]-\mathrm{RfaH}$ sample in $\mathrm{D}_{2} \mathrm{O}$ buffer ${ }^{73}$. Gradient pulses $\left(\delta_{\text {grd }} / 2\right)$ for de- and rephasing were $2.5 \mathrm{~ms}$ and the diffusion time $\left(\Delta_{\text {diff }}\right)$ was set to $80 \mathrm{~ms}$. Gradient strengths $(\mathrm{g})$ were varied between 1 and $47 \mathrm{G} \mathrm{cm}^{-1}$. The decay of signal intensity $(I)$ was fitted to Equation (2) using GraFit (Erithacus Software Ltd., Horley, UK, Version 6.0.12)

$$
\frac{I}{I_{0}}=\mathrm{e}^{-D \cdot \gamma_{\mathrm{H}}^{2} \cdot g^{2} \cdot \delta_{\mathrm{grd}}^{2}} \cdot\left(\Delta_{\text {diff }}-\frac{\delta_{\text {grd }}}{3}-\frac{\tau}{2}\right)
$$

with $I_{0}$ being the initial signal intensity, $\gamma_{\mathrm{H}}$ the gyromagnetic ratio of protons, and $\tau$ the recovery delay after the gradient pulses $(200 \mu \mathrm{s})$.

CEST experiments were carried out at $298 \mathrm{~K}$ and $900 \mathrm{MHz}{ }^{1} \mathrm{H}$-frequency according to ref. ${ }^{36}$, using a $\left[{ }^{2} \mathrm{H},{ }^{13} \mathrm{C},{ }^{15} \mathrm{~N}\right]-\mathrm{RfaH}$ sample in $25 \mathrm{mM}$ HEPES $(\mathrm{pH}$ 7.5), $50 \mathrm{mM} \mathrm{NaCl}, 5 \%\left(\mathrm{v} / \mathrm{v}\right.$ ) glycerol, $1 \mathrm{mM} \mathrm{DTT}, 10 \% \mathrm{D}_{2} \mathrm{O}$. Saturation was achieved by a $35 \mathrm{~Hz} \mathrm{~B}_{1}$-field applied during an exchange period of $500 \mathrm{~ms}$.

CPMG relaxation dispersion experiments were conducted at $288 \mathrm{~K}$ and a $700 \mathrm{MHz}{ }^{1} \mathrm{H}$-frequency using a $\left[{ }^{2} \mathrm{H},{ }^{15} \mathrm{~N}\right]$ - $\mathrm{RfaH}$ sample in $10 \mathrm{mM} \mathrm{K}_{2} \mathrm{HPO}_{4} / \mathrm{KH}_{2} \mathrm{PO}_{4}$ $(\mathrm{pH} 7.5), 50 \mathrm{mM} \mathrm{KCl}, 10 \% \mathrm{D}_{2} \mathrm{O}$. The constant time approach ${ }^{74}$ was applied with a total constant time period of $36 \mathrm{~ms}$ and $v_{\mathrm{CPMG}}$ ranging from 30 to $2000 \mathrm{~Hz}$.

Assembly of opsEC. Assembly of the ops-paused EC and design of the nucleic acids were based on published methods ${ }^{38}$. First a RNA:DNA-hybrid was formed from the ops-template (T) DNA (Supplementary Table 2) and the ops-RNA (Supplementary Table 2). Stock solutions of both oligos (1 mM in $99.9 \% \mathrm{D}_{2} \mathrm{O}$ ) were diluted with buffer (50 mM Na${ }_{2} \mathrm{HPO}_{4} / \mathrm{NaH}_{2} \mathrm{PO}_{4}$ (pH 7.5), $50 \mathrm{mM} \mathrm{KCl}, 0.3 \mathrm{mM}$ EDTA in $99.9 \% \mathrm{D}_{2} \mathrm{O}$ ) by $1: 1$ and mixed at an equimolar ratio. The mixture was incubated for $1 \mathrm{~min}$ at $95^{\circ} \mathrm{C}$, then for $10 \mathrm{~min}$ at $70^{\circ} \mathrm{C}$, and finally cooled to room temperature within $15 \mathrm{~min}$. RNAP (typically at $50-100 \mu \mathrm{M}$ ) was added at $1.3 \mathrm{molar}$ excess over the hybrid, followed by $10 \mathrm{~min}$ incubation at room temperature. Finally, the NT-opsDNA strand (Supplementary Table 2; $1 \mathrm{mM}$ stock solution in $\mathrm{D}_{2} \mathrm{O}$ ) was added at a molar ratio of 1:1.3:3 (T-ops-DNA/ops-RNA-hybrid:RNAP: NT-ops-DNA) and incubated for $10 \mathrm{~min}$ at $37^{\circ} \mathrm{C}$. To increase the long-term stability of the complex, $2 \mathrm{mM}$ DTT, $5 \mathrm{mM} \mathrm{MgCl}_{2}$ and $5 \%(\mathrm{v} / \mathrm{v}) \mathrm{d}_{8}$-glycerol were added to the sample.

In vitro transcription assay. Linear templates for in vitro transcription were made by PCR and purified via a QIAquick PCR purification kit (Qiagen, Valencia, CA). For the first-round reaction, a linear template was generated by PCR of pIA349 (Supplementary Table 3 ) using a top biotinylated primer and a bottom primer with an EcoRI recognition site, as described in ref. ${ }^{13}$. When indicated, the template was pre-incubated with a cleavage-deficient EcoRI Q111 mutant (at $3 \mu \mathrm{M}$; to ensure complete occupancy of the roadblock) in TGA2 $(20 \mathrm{mM}$ Tris-acetate, $20 \mathrm{mM} \mathrm{Na}$ acetate, $2 \mathrm{mM}$ Mg-acetate, $5 \%$ glycerol, $1 \mathrm{mM} \mathrm{DTT}, 0.1 \mathrm{mM}$ EDTA, $\mathrm{pH}$ 7.9) for $15 \mathrm{~min}$ at $37^{\circ} \mathrm{C}$. The biotinylated DNA template $(200 \mathrm{nM})$, RNAP holoenzyme $(350 \mathrm{nM}), \mathrm{ApU}(100 \mu \mathrm{M})$ and $5 \mu \mathrm{M}$ each CTP, GTP, and ATP were incubated with prewashed Streptavidin coated magnetic beads (Dynabeads ${ }^{\circledast}$ MyOneTM Streptavidin C1) in $40 \mu \mathrm{l}$ volume for $15 \mathrm{~min}$ at $37^{\circ} \mathrm{C}$ to form halted G37 ECs. WT RfaH was added at $100 \mathrm{nM}$ (to ensure that all $\mathrm{RfaH}$ was bound to the EC), followed by a 2 -min incubation at $37^{\circ} \mathrm{C}$. Unlabeled NTPs $(20 \mu \mathrm{M}$ GTP, $200 \mu \mathrm{M}$ ATP, CTP, and UTP) and rifapentin $(25 \mu \mathrm{g} / \mathrm{ml})$ were added for $10 \mathrm{~min}$ at $37^{\circ} \mathrm{C}$. The supernatant was collected using a Magnetic Separation Stand (Promega) and purified through AutoSeq G-50 spin columns (GE Healthcare) pre-equilibrated with TGA2; $25 \mu \mathrm{l} /$ column.

For the second-round reaction, WT ops (pIA1087) or G8C ops (pZL23) templates were prepared as described in ref. ${ }^{29}$. The resulting linear templates contained T7A1 promoter followed by an initial $24 \mathrm{nt}$ T-less transcribed region; the run-off transcript generated on these templates is 79-nt long. Linear DNA template $(30 \mathrm{nM})$, holo RNAP $(50 \mathrm{nM})$, ApU $(100 \mu \mathrm{M})$, and starting NTP subsets $(1 \mu \mathrm{M}$ CTP, $5 \mu \mathrm{M}$ ATP and GTP, $\left.10 \mu \mathrm{Ci}\left[\alpha^{32} \mathrm{P}\right]-\mathrm{GTP}, 3000 \mathrm{Ci} / \mathrm{mmol}\right)$ were mixed in $100 \mu \mathrm{l}$ of TGA2. Reactions were incubated for $15 \mathrm{~min}$ at $37^{\circ} \mathrm{C}$; thus halted ECs were stored on ice.

An equal volume of RfaH in TGA2 or supernatant from the first round (to yield $50 \mathrm{nM}$ final concentrations) was added to the EC, followed by a 2-min incubation at $37^{\circ} \mathrm{C}$. Transcription was restarted by addition of nucleotides $(5 \mu \mathrm{M}$ GTP, $150 \mu \mathrm{M}$ ATP, CTP, and UTP) and rifapentin to $25 \mu \mathrm{g} / \mathrm{ml}$. Samples were removed at time points indicated in the figure and quenched by addition of an equal volume of STOP buffer (10 M urea, $60 \mathrm{mM}$ EDTA, $45 \mathrm{mM}$ Tris-borate; $\mathrm{pH}$ 8.3). Samples were heated for $2 \mathrm{~min}$ at $95^{\circ} \mathrm{C}$ and separated by electrophoresis in denaturing $9 \%$ acrylamide (19:1) gels (7 M Urea, 0.5X TBE). The gels were dried and RNA products were visualized and quantified using FLA9000 Phosphorimaging System, ImageQuant Software, and Microsoft Excel. In vitro transcription assays were carried out in triplicates and averaged.

Model of $\mathbf{R f a H}^{\boldsymbol{\beta} \mathbf{C T D}}$. RfaH-NTD (PDB ID: '5OND') and RfaH-CTD in the all- $\beta$ state (PDB ID: '2LCL') were superimposed on the structure of T. maritima NusG (PDB ID: '2LQ8'). No structural rearrangements were applied.

Programs. All molecular structures were visualized using The PyMOL Molecular Graphics System (Version 1.7, Schrödinger, LLC). Superpositions of protein and nucleic-acid structures were prepared with $\mathrm{COOT}^{75}$. Interaction surfaces were analyzed by the ,protein interfaces, surfaces and assemblies' service PISA at the European Bioinformatics Institute (http://www.ebi.ac.uk/pdbe/prot_int/pistart.html) ${ }^{76}$.

\section{Data availability}

The source data underlying Fig. 2b, Fig. 4b, Fig. 5b, Fig. 6d, Supplementary Figure 2, and Supplementary Figure $5 \mathrm{e}$ are provided as a Source Data file. Other data are available form the corresponding authors upon reasonable request.

Received: 14 September 2018 Accepted: 17 January 2019

Published online: 11 February 2019

\section{References}

1. Werner, F. A nexus for gene expression-molecular mechanisms of Spt5 and NusG in the three domains of life. J. Mol. Biol. 417, 13-27 (2012).

2. Guo, S. et al. A regulator of transcriptional elongation controls vertebrate neuronal development. Nature 408, 366-369 (2000)

3. Hirtreiter, A. et al. Spt $4 / 5$ stimulates transcription elongation through the RNA polymerase clamp coiled-coil motif. Nucleic Acids Res. 38, 4040-4051 (2010).

4. Bar-Nahum, G. et al. A ratchet mechanism of transcription elongation and its control. Cell 120, 183-193 (2005).

5. Burmann, B. M. et al. A NusE:NusG complex links transcription and translation. Science 328, 501-504 (2010).

6. Lawson, M. R. et al. Mechanism for the regulated control of transcription by a universal adapter protein. Mol. Cell 71, 1-12 (2018).

7. Valabhoju, V., Agrawal, S. \& Sen, R. Molecular basis of NusG-mediated regulation of Rho-dependent transcription termination in bacteria. J. Biol. Chem. 291, 22386-22403 (2016)

8. Mandal, S. S. et al. Functional interactions of RNA-capping enzyme with factors that positively and negatively regulate promoter escape by RNA polymerase II. Proc. Natl Acad. Sci. USA 101, 7572-7577 (2004).

9. Wier, A. D., Mayekar, M. K., Héroux, A., Arndt, K. M. \& VanDemark, A. P Structural basis for Spt5-mediated recruitment of the Paf1 complex to chromatin. Proc. Natl Acad. Sci. USA 110, 17290-17295 (2013).

10. Pavri, R. et al. Activation-induced cytidine deaminase targets DNA at sites of RNA polymerase II stalling by interaction with Spt5. Cell 143, 122-133 (2010).

11. Bernecky, C., Plitzko, J. M. \& Cramer, P. Structure of a transcribing RNA polymerase II-DSIF complex reveals a multidentate DNA-RNA clamp. Nat. Struct. Mol. Biol. 24, 809-815 (2017) 
12. Ehara, H. et al. Structure of the complete elongation complex of RNA polymerase II with basal factors. Science 357, 921-924 (2017).

13. Kang, J. Y. et al. Structural basis for transcript elongation control by NusG family universal regulators. Cell 173, 1650-1662 (2018).

14. Klein, B. J. et al. RNA polymerase and transcription elongation factor Spt $4 / 5$ complex structure. Proc. Natl Acad. Sci. USA 108, 546-550 (2011).

15. Martinez-Rucobo, F. W., Sainsbury, S., Cheung, A. C. M. \& Cramer, P. Architecture of the RNA polymerase-Spt $4 / 5$ complex and basis of universal transcription processivity. EMBO J. 30, 1302-1310 (2011).

16. Kyrpides, N. C., Woese, C. R. \& Ouzounis, C. A. KOW: a novel motif linking a bacterial transcription factor with ribosomal proteins. Trends Biochem. Sci. 21, 425-426 (1996).

17. Peters, J. M. et al. Rho and NusG suppress pervasive antisense transcription in Escherichia coli. Genes Dev. 26, 2621-2633 (2012).

18. Saxena, S. et al. Escherichia coli transcription factor NusG binds to $70 \mathrm{~S}$ ribosomes. Mol. Microbiol. 108, 495-504 (2018).

19. Mayer, A. et al. Uniform transitions of the general RNA polymerase II transcription complex. Nat. Struct. Mol. Biol. 17, 1272-1278 (2010).

20. Mooney, R. A. et al. Regulator trafficking on bacterial transcription units in vivo. Mol. Cell 33, 97-108 (2009).

21. Rahl, P. B. et al. c-Myc regulates transcriptional pause release. Cell 141, 432-445 (2010).

22. Gruchota, J., Denby Wilkes, C., Arnaiz, O., Sperling, L. \& Nowak, J. K. A meiosis-specific Spt5 homolog involved in non-coding transcription. Nucleic Acids Res. 45, 4722-4732 (2017).

23. Bies-Etheve, N. et al. RNA-directed DNA methylation requires an AGO4interacting member of the SPT5 elongation factor family. EMBO Rep. 10, 649-654 (2009).

24. Goodson, J. R., Klupt, S., Zhang, C., Straight, P. \& Winkler, W. C. LoaP is a broadly conserved antiterminator protein that regulates antibiotic gene clusters in Bacillus amyloliquefaciens. Nat. Microbiol. 2, 17003-17012 (2017).

25. NandyMazumdar, M. \& Artsimovitch, I. Ubiquitous transcription factors display structural plasticity and diverse functions: NusG proteins-shifting shapes and paradigms. Bioessays 37, 324-334 (2015).

26. Sevostyanova, A., Belogurov, G. A., Mooney, R. A., Landick, R. \& Artsimovitch, I. The $\beta$ subunit gate loop is required for RNA polymerase modification by RfaH and NusG. Mol. Cell 43, 253-262 (2011).

27. $\mathrm{Hu}, \mathrm{K}$. \& Artsimovitch, I. A Screen for $r f a H$ suppressors reveals a key role for a connector region of termination factor Rho. MBio 8, https://doi.org/10.1128/ mBio.00753-17 (2017).

28. Belogurov, G. A., Mooney, R. A., Svetlov, V., Landick, R. \& Artsimovitch, I. Functional specialization of transcription elongation factors. EMBO J. 28, 112-122 (2009).

29. Zuber, P. K. et al. The universally-conserved transcription factor RfaH is recruited to a hairpin structure of the non-template DNA strand. eLlife 7 , e36349 (2018).

30. Belogurov, G. A. et al. Structural basis for converting a general transcription factor into an operon-specific virulence regulator. Mol. Cell 26, 117-129 (2007).

31. Burmann, B. M. et al. An $\alpha$ helix to $\beta$ barrel domain switch transforms the transcription factor RfaH into a translation factor. Cell 150, 291-303 (2012).

32. Tomar, S. K., Knauer, S. H., Nandymazumdar, M., Rösch, P. \& Artsimovitch, I. Interdomain contacts control folding of transcription factor RfaH. Nucleic Acids Res. 41, 10077-10085 (2013).

33. Shi, D., Svetlov, D., Abagyan, R. \& Artsimovitch, I. Flipping states: a few key residues decide the winning conformation of the only universally conserved transcription factor. Nucleic Acids Res. 45, 8835-8843 (2017).

34. Knauer, S. H., Artsimovitch, I. \& Rösch, P. Transformer proteins. Cell Cycle 11, 4289-4290 (2012).

35. Sprangers, R., Velyvis, A. \& Kay, L. E. Solution NMR of supramolecular complexes: providing new insights into function. Nat. Methods 4, 697-703 (2007).

36. Vallurupalli, P., Bouvignies, G. \& Kay, L. E. Studying 'invisible' excited protein states in slow exchange with a major state conformation. J. Am. Chem. Soc. 134, 8148-8161 (2012)

37. Mittermaier, A. \& Kay, L. E. New tools provide new insights in NMR studies of protein dynamics. Science 312, 224-228 (2006).

38. Artsimovitch, I. \& Landick, R. The transcriptional regulator RfaH stimulates RNA chain synthesis after recruitment to elongation complexes by the exposed nontemplate DNA strand. Cell 109, 193-203 (2002).

39. Drögemüller, J. et al. Determination of RNA polymerase binding surfaces of transcription factors by NMR spectroscopy. Sci. Rep. 5, 16428-16441 (2015).

40. Luo, X. et al. Structural and functional analysis of the E. coliNusB-S10 transcription antitermination complex. Mol. Cell 32, 791-802 (2008).

41. Ramírez-Sarmiento, C. A., Noel, J. K., Valenzuela, S. L. \& Artsimovitch, I. Interdomain contacts control native state switching of RfaH on a dualfunneled landscape. PLoS Comput. Biol. 11, https://doi.org/10.1371/journal. pcbi.1004379 (2015).
42. Nedialkov, Y., Svetlov, D., Belogurov, G. A. \& Artsimovitch, I. Locking the non-template DNA to control transcription. Mol. Microbiol. 109, 445-457 (2018).

43. Mooney, R. A., Schweimer, K., Rösch, P., Gottesman, M. \& Landick, R. Two structurally independent domains of E. coli NusG create regulatory plasticity via distinct interactions with RNA polymerase and regulators. J. Mol. Biol. 391, 341-358 (2009).

44. Pufall, M. A. \& Graves, B. J. Autoinhibitory domains: modular effectors of cellular regulation. Annu. Rev. Cell. Dev. Biol. 18, 421-462 (2002).

45. Currie, S. L. et al. Structured and disordered regions cooperatively mediate DNA-binding autoinhibition of ETS factors ETV1, ETV4 and ETV5. Nucleic Acids Res. 45, 2223-2241 (2017).

46. Au-Yeung, B. B., Shah, N. H., Shen, L. \& Weiss, A. ZAP-70 in signaling, biology, and disease. Annu. Rev. Immunol. 36, 127-156 (2018).

47. Cai, Z. et al. Fatty acid DSF binds and allosterically activates histidine kinase $\mathrm{RpfC}$ of phytopathogenic bacterium Xanthomonas campestris pv. campestris to regulate quorum-sensing and virulence. PLoS Pathog. 13, https://doi.org/ 10.1371/journal.ppat.1006304 (2017).

48. Takemoto-Kimura, S. et al. Calmodulin kinases: essential regulators in health and disease. J. Neurochem. 141, 808-818 (2017).

49. Buetow, L. \& Huang, D. T. Structural insights into the catalysis and regulation of E3 ubiquitin ligases. Nat. Rev. Mol. Cell Biol. 17, 626-642 (2016).

50. Mitra, P., Ghosh, G., Hafeezunnisa, M. \& Sen, R. Rho protein: roles and mechanisms. Annu. Rev. Microbiol. 71, 687-709 (2017).

51. Schmidt, A. et al. The quantitative and condition-dependent Escherichia coli proteome. Nat. Biotechnol. 34, 104-110 (2016).

52. Burmann, B. M., Scheckenhofer, U., Schweimer, K. \& Rösch, P. Domain interactions of the transcription-translation coupling factor Escherichia coli NusG are intermolecular and transient. Biochem. J. 435, 783-789 (2011).

53. Drögemüller, J. et al. An autoinhibited state in the structure of Thermotoga maritima NusG. Structure 21, 365-375 (2013).

54. Drögemüller, J. et al. Thermotoga maritima NusG: domain interaction mediates autoinhibition and thermostability. Nucleic Acids Res. 45, 446-460 (2017).

55. Anfinsen, C. B. Principles that govern the folding of protein chains. Science 181, 223-230 (1973).

56. Murzin, A. G. Biochemistry. Metamorphic proteins. Science 320, 1725-1726 (2008).

57. Dishman, A. F. \& Volkman, B. F. Unfolding the mysteries of protein metamorphosis. Acs. Chem. Biol. 13, 1438-1446 (2018).

58. Awasthi, S. K., Shankaramma, S. C., Raghothama, S. \& Balaram, P. Solventinduced beta-hairpin to helix conformational transition in a designed peptide. Biopolymers 58, 465-476 (2001).

59. Cerpa, R., Cohen, F. E. \& Kuntz, I. D. Conformational switching in designed peptides: the helix/sheet transition. Fold. Des. 1, 91-101 (1996).

60. Newlove, T., Konieczka, J. H. \& Cordes, M. H. J. Secondary structure switching in Cro protein evolution. Structure 12, 569-581 (2004).

61. Tuinstra, R. L. et al. Interconversion between two unrelated protein folds in the lymphotactin native state. Proc. Natl Acad. Sci. USA 105, 5057-5062 (2008).

62. Mueller, M., Grauschopf, U., Maier, T., Glockshuber, R. \& Ban, N. The structure of a cytolytic alpha-helical toxin pore reveals its assembly mechanism. Nature 459, 726-730 (2009).

63. Chang, Y.-G. et al. Circadian rhythms. A protein fold switch joins the circadian oscillator to clock output in cyanobacteria. Science 349, 324-328 (2015).

64. Burgie, E. S., Bussell, A. N., Walker, J. M., Dubiel, K. \& Vierstra, R. D. Crystal structure of the photosensing module from a red/far-red light-absorbing plant phytochrome. Proc. Natl Acad. Sci. USA 111, 10179-10184 (2014).

65. Zamora-Carreras, H. et al. Micelle-triggered $\beta$-hairpin to $\alpha$-helix transition in a 14-residue peptide from a choline-binding repeat of the pneumococcal autolysin LytA. Chemistry 21, 8076-8089 (2015).

66. Surewicz, W. K. \& Apostol, M. I. Prion protein and its conformational conversion: a structural perspective. Top. Curr. Chem. 305, 135-167 (2011).

67. Burmann, B. M., Luo, X., Rösch, P., Wahl, M. C. \& Gottesman, M. E. Fine tuning of the E. coli NusB:NusE complex affinity to BoxA RNA is required for processive antitermination. Nucleic Acids Res. 38, 314-326 (2010).

68. Svetlov, V. \& Artsimovitch, I. Purification of bacterial RNA polymerase: tools and protocols. Methods Mol. Biol. 1276, 13-29 (2015).

69. Drögemüller, J. et al. Exploring RNA polymerase regulation by NMR spectroscopy. Sci. Rep. 5, 10825-10835 (2015).

70. Meyer, O. \& Schlegel, H. G. Biology of aerobic carbon monoxide-oxidizing bacteria. Annu. Rev. Microbiol. 37, 277-310 (1983).

71. Sambrook, J. \& Russel, D. W. Molecular Cloning: A Laboratory Manual Vol. 3 (Cold Spring Harbor Press, 2001).

72. Sprangers, R. \& Kay, L. E. Quantitative dynamics and binding studies of the $20 S$ proteasome by NMR. Nature 445, 618-622 (2007). 
73. Didenko, T., Boelens, R. \& Rüdiger, S. G. D. 3D DOSY-TROSY to determine the translational diffusion coefficient of large protein complexes. Protein Eng. Des. Sel. 24, 99-103 (2011)

74. Tollinger, M., Skrynnikov, N. R., Mulder, F. A., Forman-Kay, J. D. \& Kay, L. E. Slow dynamics in folded and unfolded states of an SH3 domain. J. Am. Chem. Soc. 123, 11341-11352 (2001).

75. Emsley, P., Lohkamp, B., Scott, W. G. \& Cowtan, K. Features and development of Coot. Acta Crystallogr. D. Biol. Crystallogr. 66, 486-501 (2010).

76. Krissinel, E. \& Henrick, K. Inference of macromolecular assemblies from crystalline state. J. Mol. Biol. 372, 774-797 (2007).

\section{Acknowledgements}

We thank Ramona Heißmann, and Andrea Hager for excellent technical assistance. P.K.Z. was supported by the The Elite Network Bavaria in the framework of the Elite Study Program "Macromolecular Science". The work was supported by grant Ro 617/21-1 (P.R.) from the Deutsche Forschungsgemeinschaft, the Ludwig-Schaefer award of Columbia University (P.R.), and grant GM67153 (I.A.) from the National Institutes of Health.

\section{Author contributions}

P.K.Z., K.S., and S.H.K. carried out the NMR experiments. I.A. carried out the in vitro experiments. S.H.K., P.R., and I.A. designed and supervised research and prepared the manuscript with input from all authors.

\section{Additional information}

Supplementary Information accompanies this paper at https://doi.org/10.1038/s41467019-08567-6.
Competing interests: The authors declare no competing interests.

Reprints and permission information is available online at http://npg.nature.com/ reprintsandpermissions/

Journal peer review information: Nature Communications thanks the anonymous reviewers for their contribution to the peer review of this work. Peer reviewer reports are available.

Publisher's note: Springer Nature remains neutral with regard to jurisdictional claims in published maps and institutional affiliations.

(c) (i) Open Access This article is licensed under a Creative Commons Attribution 4.0 International License, which permits use, sharing, adaptation, distribution and reproduction in any medium or format, as long as you give appropriate credit to the original author(s) and the source, provide a link to the Creative Commons license, and indicate if changes were made. The images or other third party material in this article are included in the article's Creative Commons license, unless indicated otherwise in a credit line to the material. If material is not included in the article's Creative Commons license and your intended use is not permitted by statutory regulation or exceeds the permitted use, you will need to obtain permission directly from the copyright holder. To view a copy of this license, visit http://creativecommons.org/ licenses/by/4.0/.

(C) The Author(s) 2019 